\title{
A Review of Some Recent Results for the Approximate Analytical Solutions of Nonlinear Differential Equations
}

\author{
Serdal Pamuk \\ Department of Mathematics, University of Kocaeli, Umuttepe, 41800 Kocaeli, Turkey \\ Correspondence should be addressed to Serdal Pamuk, spamuk@kocaeli.edu.tr \\ Received 15 January 2009; Revised 21 April 2009; Accepted 29 April 2009 \\ Recommended by Ji Huan He \\ This paper features a survey of some recent developments in techniques for obtaining approximate \\ analytical solutions of some nonlinear differential equations arising in various fields of science \\ and engineering. Adomian's decomposition method is applied to some nonlinear problems, and \\ some mathematical tools such as He's homotopy perturbation method and variational iteration \\ method are introduced to overcome the shortcomings of Adomian's method. The results of some \\ comparisons of these three methods appearing in the research literature are given. \\ Copyright (C) 2009 Serdal Pamuk. This is an open access article distributed under the Creative \\ Commons Attribution License, which permits unrestricted use, distribution, and reproduction in \\ any medium, provided the original work is properly cited.
}

\section{Introduction}

Nonlinear phenomena play a crucial role in applied mathematics and engineering. Therefore, over the last ten years, so many mathematical methods that are aimed at obtaining analytical solutions of nonlinear differential equations arising in various fields of science and engineering have appeared in the research literature [1-6]. However, most of them require a tedious analysis or a large computer memory to handle these problems.

In this paper we present and compare three methods which are recently studied by the scientists to obtain approximate analytical solutions of some nonlinear differential equations arising in various fields of science and engineering.

The first method is so-called Adomian decomposition method (ADM) which was introduced by Adomian [7-13] in the beginning of the 1980s. This is an iterative method which provides approximate analytical solutions in the form of an infinite power series for nonlinear equations. It is well known that this method avoids linearization, discretization and scientifically unrealistic assumptions. It also provides an efficient numerical solution with high accuracy $[6,7,14]$. This method is modified and used by Jin and Liu [15] to improve the 
convergence of series solution. They apply the modified ADM to solve a kind of evolution equations. Also, the authors of [16-18] apply the ADM to obtain the approximate analytical solutions for heat-like and wave-like equations with variable coefficients, for the wave equation in an infinite one-dimensional medium and for Bratu-type equations, respectively.

The second method is the homotopy perturbation method (HPM) which was proposed by He [19] in 1999. In this method, the solution is obtained as the summation of an infinite series, which converges to analytical solution. Using the homotopy technique from topology, a homotopy is constructed with an embedding parameter $p \in[0,1]$, which is considered as a "small parameter". The approximations obtained by the HPM are uniformly valid not only for small parameters but also for very large parameters. Also, this method is modified and used by some scientists to obtain a fast convergent rate (see, e.g., [20]).

The last method is the variational iteration method (VIM) which is based on the incorporation of a general Lagrange multiplier in the construction of correction functional for the equation. This method has been proposed by Shou and He [21] and is thoroughly used by many researchers (see, e.g., [22-26]) to handle linear and nonlinear problems. The VIM uses only the prescribed initial conditions and does not require a specific treatment.

Although it is revealed that modified form of HPM corresponds to ADM for certain nonlinear problems [27], many researchers find ADM very difficult to calculate the Adomian polynomials [23, 28-31]. Also, ADM could not always satisfy all the boundary conditions of the nonlinear problems, leading to an error at the boundary of the domain in which the problem is solved [32].

On the other hand, the authors of $[33,34]$ overcome the shortcomings of the Adomian method using HPM and He polynomials, and they state that HPM and He polynomials can completely replace the Adomian method and Adomian polynomials.

Compared with Adomian method, HPM and He polynomials do have some obvious merits: (1) the method needs not to calculate Adomian polynomials; (2) the method is very straightforward, and the solution procedure is very simple [20, 24-26, 35-37].

In their calculations of the analytical solutions of various kinds of heat-like and wavelike equations, the authors of [21] pointed out that contrary to Adomian method, VIM needs no calculation of Adomian polynomial, only simple operation is needed. Another nice comparison between ADM and VIM is given by Wazwaz [38]. In his study he concludes the following: VIM gives several successive approximations through using the iteration of the correction functional. However, ADM provides the components of the exact solution, where these components should follow the summation of an infinite power series. Moreover, the VIM requires the evaluation of the Lagrangian multiplier $\lambda$, whereas ADM requires the evaluation of the Adomian polynomials that mostly require tedious algebraic calculations. More importantly, the VIM reduces the volume of calculations by not requiring the Adomian polynomials, hence the iteration is direct and straightforward. However, ADM requires the use of Adomian polynomials for nonlinear terms, and this needs more work. For nonlinear equations that arise frequently to express nonlinear phenomenon, He's VIM facilitates the computational work and gives the solution rapidly if compared with ADM.

Hojjati and Jafari [39] have made a comparison among these three methods, and they have concluded that although the numerical results are almost the same, HPM is much easier, more convenient and efficient than ADM and VIM.

In [40], the author features a survey of some recent developments in asymptotic technics, which are valid not only for weakly nonlinear equations but also for strongly ones. The limitations of the traditional perturbation methods are illustrated, various modified perturbation techniques are proposed, and some mathematical tools such as variational 
theory, homotopy technology, and iteration technique are introduced to overcome the shortcomings. In [41], the author pays particular attention throughout the paper to give an intuitive grasp for Lagrange multiplier, calculus of variations, optimization, VIM, parameterexpansion method, exp-function method, HPM, and ancient Chinese mathematics as well. Subsequently, nanomechanics in textile engineering and E-infinity theory in high-energy physics, Kleiber's 3/4 law in biology, possible mechanism in spider-spinning process, and fractal approach to carbon nanotube are briefly introduced. In [42], the same author presents a coupling method of a homotopy technique and a perturbation technique to solve nonlinear problems. In contrast to traditional perturbation methods, HPM does not require a small parameter in the equation.

We now present some of the equations from our last work [3-5] related to nonlinear problems of various fields of science and engineering.

First, we consider the logistic growth in a population as a single species model to be governed by [43]

$$
\frac{d N}{d t}=r N(1-N / K)
$$

where $r$ and $K$ are positive constants. Here $N=N(t)$ represents the population of the species at time $t, r(1-N / K)$ is the per capita growth rate, and $K$ is the carrying capacity of the environment. We nondimensionalize (1.1) by setting

$$
u(\tau)=\frac{N(t)}{K}, \quad \tau=r t
$$

and it becomes

$$
\frac{d u}{d \tau}=u(1-u)
$$

If $N(0)=N_{0}$, then $u(0)=N_{0} / K$. Therefore, the analytical solution of $(1.3)$ is easily obtained:

$$
u(\tau)=\frac{1}{1+\left(K / N_{0}-1\right) e^{-\tau}} .
$$

Second, we consider the Predator-Prey Models: Lotka-Volterra systems as an interacting species model to be governed by $[3,43,44]$

$$
\begin{aligned}
& \frac{d N}{d t}=N(a-b P), \\
& \frac{d P}{d t}=P(c N-d),
\end{aligned}
$$


where $a, b, c$, and $d$ are constants. Here $N=N(t)$ is the prey population and $P=P(t)$ that of the predator at time $t$. We nondimensionalize the system (1.5) [43] by setting

$$
u(\tau)=\frac{c N(t)}{d}, \quad v(\tau)=\frac{b P(t)}{a}, \quad \tau=a t, \alpha=d / a,
$$

and it becomes

$$
\begin{aligned}
& \frac{d u}{d \tau}=u(1-v), \\
& \frac{d v}{d \tau}=\alpha v(u-1) .
\end{aligned}
$$

Third, we present the heat equation [4]:

$$
u_{t}=u_{x x}+\epsilon u^{m}
$$

where $m=1,2,3, \ldots$, and $\epsilon$ is a parameter. Here, the indices $t$ and $x$ denote derivatives with respect to these variables. Unless $m=1,(1.8)$ is a nonlinear heat equation. Construction of particular analytical solutions for nonlinear equations of the form (1.8) is an important problem. Especially, finding an analytical solution that has a biological interpretation is of fundamental importance. Recently, some new methods such as Lie symmetry reduction method [45], and antireduction method [46] which transforms the nonlinear PDEs to a system of ODEs have been introduced in the research literature to find particular analytical solutions to PDE. Finding analytical solutions of most nonlinear PDE generally requires new methods. form

The particular analytical solutions of the nonlinear reaction diffusion equations of the

$$
u_{t}=\left(A(u) u_{x}\right)_{x}+B(u) u_{x}+C(u)
$$

where $A(u), B(u)$, and $C(u)$ are specially chosen smooth functions, are obtained in [47]. This equation usually arises in mathematical biology $[43,44]$. In fact, $(1.8)$ is a particular case of the last equation.

We last consider the nonlinear heat equation called the porous media equation [5]:

$$
\frac{\partial u}{\partial t}=\frac{\partial}{\partial x}\left(u^{m} \frac{\partial u}{\partial x}\right)
$$

where $m$ is a rational number.

Finding the particular analytical solutions that have a physical or biological interpretation for the nonlinear equations of the form (1.10) is of fundamental importance. This equation often occurs in nonlinear problems of heat and mass transfer, combustion theory, and flows in porous media. For example, it describes unsteady heat transfer in a quiescent medium with the heat diffusivity being a power-law function of temperature [48]. 
Equation (1.10) has also applications to many physical systems including the fluid dynamics of thin films [49]. Murray [43] describes how this model has been used to represent "population pressure" in biological systems. This equation is called a degenerate parabolic differential equation because the diffusion coefficient $D(u)=u^{m}$ does not satisfy the condition for classical diffusion equations, $D(u)>0$ [49]. For the motion of thin viscous films, (1.10) with $m=3$ can be derived from the Navier-Stokes equations. Lacking a physical law to describe the complex behavior in a system, an appropriate value for the parameter $m$ can be determined by comparing known solutions with empirical data [49].

In the following section, we apply the ADM [7-13] to (1.3), (1.7)-(1.10), respectively.

\section{Adomian's Decomposition Method}

\subsection{Analysis of the Method for Single Species}

In this section we consider the model equation of the form [3]

$$
\frac{d u}{d \tau}=u-f(u), \quad u(0)=r,
$$

where $f$ is a nonlinear function of $u$. We are looking for the solution $u$ satisfying (2.1). The decomposition method consists of approximating the solution of (2.1) as an infinite series:

$$
u=\sum_{n=0}^{\infty} u_{n}
$$

and decomposing $f$ as

$$
f(u)=\sum_{n=0}^{\infty} A_{n}
$$

where $A_{n}$ 's are the Adomian polynomials given by

$$
A_{n}=\frac{1}{n !} \frac{d^{n}}{d \lambda^{n}}\left[f\left(\sum_{n=0}^{\infty} \lambda^{n} u_{n}\right)\right]_{\lambda=0}, \quad n=0,1,2, \ldots
$$

The convergence of the decomposition series (2.3) is studied in [50]. Applying the decomposition method $[7,14],(2.1)$ can be written as

$$
L u=u-f(u),
$$

where the notation $L=\partial / \partial \tau$ symbolizes the linear differential operator. We assume the integration inverse operators $L^{-1}$ exist, and it is defined as $L^{-1}=\int_{0}^{\tau}(\cdot) d \tau$. Therefore, applying on both sides of (2.5) with $L^{-1}$ yields

$$
u(\tau)=u(0)+L^{-1} u(\tau)-L^{-1} f(u(\tau)) .
$$


Using (2.2) and (2.3), it follows that

$$
\sum_{n=0}^{\infty} u_{n}=u(0)+L^{-1} \sum_{n=0}^{\infty} u_{n}-L^{-1} \sum_{n=0}^{\infty} A_{n}
$$

Therefore, one determines the iterates in the following recursive way:

$$
\begin{gathered}
u_{0}=u(0)=r, \\
u_{n+1}=L^{-1} u_{n}-L^{-1} A_{n}, \quad n=0,1,2, \ldots
\end{gathered}
$$

We then define the solution $u$ as

$$
u=\lim _{n \rightarrow \infty} \sum_{k=0}^{n} u_{k}
$$

\subsection{Analysis of the Method for Interacting Species}

In this section, we consider the system of the form [3]

$$
\begin{aligned}
& \frac{d u}{d \tau}=u-f(u, v) \\
& \frac{d v}{d \tau}=\alpha[g(u, v)-v]
\end{aligned}
$$

with initial data

$$
u(0)=\delta, \quad v(0)=\beta
$$

Here, $f$ and $g$ are nonlinear functions of $u$ and $v$. We are looking for the solutions $(u, v)$ satisfying (2.10)-(2.11). The decomposition method consists of approximating the solutions of the above system as an infinite series:

$$
u=\sum_{n=0}^{\infty} u_{n}, \quad v=\sum_{n=0}^{\infty} v_{n}
$$

and decomposing $f$ and $g$ as [6]

$$
f(u, v)=\sum_{n=0}^{\infty} B_{n}, \quad g(u, v)=\sum_{n=0}^{\infty} C_{n},
$$


where $B_{n}$ and $C_{n}$ are the Adomian polynomials that can be generated for any form of nonlinearity. Applying the decomposition method, the system (2.10) can be written as

$$
\begin{aligned}
& L u=u-f(u, v), \\
& L v=\alpha[g(u, v)-v],
\end{aligned}
$$

where the notation $L=\partial / \partial \tau$ again symbolizes the linear differential operator. Therefore, applying on both sides of the equations of the system (2.14) with $L^{-1}$ yields [6]

$$
\begin{aligned}
& u(\tau)=u(0)+L^{-1} u(\tau)-L^{-1} f(u(\tau), v(\tau)), \\
& v(\tau)=v(0)+\alpha\left[L^{-1} g(u(\tau), v(\tau))-L^{-1} v(\tau)\right] .
\end{aligned}
$$

Using (2.12) and (2.13), it follows that

$$
\begin{aligned}
& \sum_{n=0}^{\infty} u_{n}=u(0)+L^{-1} \sum_{n=0}^{\infty} u_{n}-L^{-1} \sum_{n=0}^{\infty} B_{n} \\
& \sum_{n=0}^{\infty} v_{n}=v(0)+\alpha\left[L^{-1} \sum_{n=0}^{\infty} C_{n}-L^{-1} \sum_{n=0}^{\infty} v_{n}\right] .
\end{aligned}
$$

Therefore, one determines the iterates in the following recursive way:

$$
\begin{aligned}
u_{0} & =u(0)=\delta, \\
u_{n+1} & =L^{-1} u_{n}-L^{-1} B_{n}, \quad n=0,1,2, \ldots, \\
v_{0} & =v(0)=\beta, \\
v_{n+1} & =\alpha\left[L^{-1} C_{n}-L^{-1} v_{n}\right], \quad n=0,1,2 \ldots
\end{aligned}
$$

We then define the solutions of the initial value problem (2.10)-(2.11) as

$$
(u, v)=\left(\lim _{n \rightarrow \infty} \sum_{k=0}^{n} u_{k}, \lim _{n \rightarrow \infty} \sum_{k=0}^{n} v_{k}\right) .
$$

\subsection{Analysis of the Method for the Heat Equation $u_{t}=u_{x x}+\epsilon u^{m}$}

In this section, we consider (1.8) in an operator form [4]

$$
L_{t}(u(x, t))-L_{x}(u(x, t))-\epsilon u^{m}=0,
$$


Table 1: Numerical results (in $t$ direction) for $\left|u(x, t)-\phi_{30}(x, t)\right|$ where $u(x, t)=e^{\left(1-\pi^{2}\right) t} \cos (\pi x)$ for (3.12)(3.14).

\begin{tabular}{lccccc}
\hline$t_{i} \mid x_{i}$ & 0.1 & 0.2 & 0.3 & 0.4 & 0.5 \\
\hline 0.1 & $1.9942 E-21$ & $1.6973 E-21$ & $1.2322 E-21$ & $6.4790 E-22$ & $0.0000 E+00$ \\
0.2 & $4.3765 E-20$ & $3.7228 E-20$ & $2.7048 E-20$ & $1.4220 E-20$ & $0.0000 E+00$ \\
0.3 & $8.5955 E-20$ & $7.3116 E-20$ & $5.3123 E-20$ & $2.7928 E-20$ & $0.0000 E+00$ \\
0.4 & $1.0148 E-16$ & $8.6329 E-17$ & $6.2722 E-17$ & $3.2975 E-17$ & $0.0000 E+00$ \\
0.5 & $7.9889 E-14$ & $6.7958 E-14$ & $4.9374 E-14$ & $2.5957 E-14$ & $0.0000 E+00$ \\
\hline
\end{tabular}

with the initial and boundary conditions, where the notations $L_{t}=\partial / \partial t$ and $L_{x}=\partial^{2} / \partial x^{2}$ symbolize the linear differential operators. We assume the integration inverse operators $L_{t}^{-1}$ and $L_{x}{ }^{-1}$ exist, and they are defined as $L_{t}^{-1}=\int_{0}^{t}(\cdot) d t$ and $L_{x}{ }^{-1}=\iint_{0}^{x}(\cdot) d x d x$, respectively. Therefore, we can write the solutions in $t$ and $x$ directions as $[1,2,7]$

$$
\begin{aligned}
& u(x, t)=u(x, 0)+L_{t}^{-1}\left[L_{x}(u(x, t))+\Phi(u)\right], \\
& u(x, t)=u(0, t)+x u_{x}(0, t)+L_{x}{ }^{-1}\left[L_{t}(u(x, t))-\Phi(u)\right],
\end{aligned}
$$

respectively, where $\Phi(u)=\epsilon u^{m}$. By ADM [7], one can write the solution in series form as

$$
u(x, t)=\sum_{n=0}^{\infty} u_{n}(x, t)
$$

To find the solutions in $t$ and $x$ directions, one solves the recursive relations:

$$
\begin{gathered}
u_{0}=u(x, 0), \quad u_{n+1}=L_{t}^{-1}\left[L_{x}\left(u_{n}\right)+A_{n}\right], \quad n \geq 0, \\
u_{0}=u(0, t)+x u_{x}(0, t), \quad u_{n+1}=L_{x}{ }^{-1}\left[L_{t}\left(u_{n}\right)-A_{n}\right], \quad n \geq 0,
\end{gathered}
$$

respectively, where the Adomian polynomials are $[1,2,7]$

$$
A_{n}=\frac{1}{n !} \frac{d^{n}}{d \lambda^{n}}\left[\Phi\left(\sum_{n=0}^{\infty} \lambda^{n} u_{n}\right)\right]_{\lambda=0}, \quad n \geq 0
$$

We obtain the first few Adomian polynomials for $\Phi(u)=\epsilon u^{m}$ as $A_{0}=\epsilon \mathcal{u}_{0}^{m}, A_{1}=m \epsilon u_{0}^{m-1} u_{1}$, $A_{2}=(m \epsilon / 2)\left[(m-1) u_{0}^{m-2} u_{1}^{2}+2 u_{2} u_{0}^{m-1}\right]$, and so on. The convergence of the decomposition series given by (2.21) is studied in [50].

In Section 3, we provide a couple of examples and demonstrate the absolute errors $\left|u(x, t)-\phi_{n}(x, t)\right|$ in Tables $1-4$, where $u(x, t)$ is the particular analytical solution and $\phi_{n}(x, t)$ is the partial sum:

$$
\phi_{n}(x, t)=\sum_{k=0}^{n} u_{k}(x, t), \quad n \geq 0 .
$$


Table 2: Numerical results (in $x$ direction) for $\left|u(x, t)-\phi_{30}(x, t)\right|$ where $u(x, t)=e^{\left(1-\pi^{2}\right) t} \cos (\pi x)$ for (3.12)(3.14).

\begin{tabular}{lccccc}
\hline$t_{i} \mid x_{i}$ & 0.1 & 0.2 & 0.3 & 0.4 & 0.5 \\
\hline 0.1 & $4.5975 E-21$ & $3.9198 E-21$ & $5.5044 E-21$ & $4.8426 E-21$ & $2.9253 E-20$ \\
0.2 & $2.0957 E-21$ & $4.4964 E-21$ & $2.5076 E-21$ & $2.7629 E-22$ & $3.1241 E-22$ \\
0.3 & $2.7394 E-22$ & $3.5343 E-22$ & $6.4007 E-22$ & $4.6883 E-22$ & $3.0307 E-21$ \\
0.4 & $2.2349 E-22$ & $9.1141 E-23$ & $4.2382 E-22$ & $1.9278 E-22$ & $5.7223 E-22$ \\
0.5 & $1.1388 E-23$ & $9.3840 E-23$ & $2.0905 E-22$ & $2.9358 E-22$ & $6.2591 E-22$ \\
\hline
\end{tabular}

As it is clear from (2.21) and (2.25), we have

$$
u(x, t)=\lim _{n \rightarrow \infty} \phi_{n}(x, t) .
$$

\subsection{Analysis of the Method for the Porous Media Equation}

Equation (1.10) can be written in an operator form [5]

$$
L_{t}(u)=L_{x}\left(u^{m} L_{x} u\right)
$$

with the initial and boundary conditions, where the notations $L_{t}=\partial / \partial t$ and $L_{x}=\partial / \partial x$ symbolize the linear differential operators. We assume the integration inverse operators $L_{t}^{-1}$ and $L_{x}{ }^{-1}$ exist, and they are defined as $L_{t}{ }^{-1}=\int_{0}^{t}(\cdot) d t$ and $L_{x}{ }^{-1}=\int_{0}^{x}(\cdot) d x$, respectively. Therefore, one can write the solution in $t$ direction as [7]

$$
u(x, t)=u(x, 0)+L_{t}^{-1}\left[L_{x}(\Phi(u))\right]
$$

where $\Phi(u)=u^{m} u_{x}$. By ADM [7] one can write the solution in series form as

$$
u(x, t)=\sum_{n=0}^{\infty} u_{n}(x, t)
$$

To find the solutions in $t$ direction, one solves the recursive relations:

$$
u_{0}=u(x, 0), \quad u_{n+1}=L_{t}^{-1}\left[L_{x}\left(A_{n}\right)\right], \quad n \geq 0,
$$

respectively, where the Adomian polynomials are $[1,2,7]$

$$
A_{n}=\frac{1}{n !} \frac{d^{n}}{d \lambda^{n}}\left[\Phi\left(\sum_{n=0}^{\infty} \lambda^{n} u_{n}\right)\right]_{\lambda=0}, \quad n \geq 0
$$


Table 3: Numerical results (in $t$ direction) for $\left|u(x, t)-\phi_{20}(x, t)\right|$ where $u(x, t)=(1+2 x) /\left(x^{2}+x+6 t+1\right)$ for (3.19), (3.24)-(3.25).

\begin{tabular}{lccccc}
\hline$t_{i} \mid x_{i}$ & 0.01 & 0.02 & 0.03 & 0.04 & 0.05 \\
\hline 0.01 & $3.0000 E-20$ & $4.0000 E-20$ & $3.0000 E-20$ & $2.0000 E-20$ & $1.0000 E-20$ \\
0.02 & $2.8000 E-19$ & $2.8000 E-19$ & $1.7000 E-19$ & $1.4000 E-19$ & $1.3000 E-19$ \\
0.03 & $8.9367 E-16$ & $7.3753 E-16$ & $6.0717 E-16$ & $4.9881 E-16$ & $4.0888 E-16$ \\
0.04 & $2.6828 E-13$ & $2.2148 E-13$ & $1.8242 E-13$ & $1.4992 E-13$ & $1.2295 E-13$ \\
0.05 & $2.2204 E-11$ & $1.8337 E-11$ & $1.5109 E-11$ & $1.2422 E-11$ & $1.0191 E-11$ \\
\hline
\end{tabular}

Table 4: Numerical results (in $x$ direction) for $\left|u(x, t)-\phi_{20}(x, t)\right|$ where $u(x, t)=(1+2 x) /\left(x^{2}+x+6 t+1\right)$ for (3.19), (3.24)-(3.25).

\begin{tabular}{lccccc}
\hline$t_{i} \mid x_{i}$ & 0.01 & 0.02 & 0.03 & 0.04 & 0.05 \\
\hline 0.01 & $1.0000 E-20$ & $1.0000 E-20$ & $1.0000 E-20$ & $1.0000 E-20$ & $1.0000 E-20$ \\
0.02 & $1.0000 E-20$ & $1.0000 E-20$ & $0.0000 E+00$ & $1.0000 E-20$ & $0.0000 E+00$ \\
0.03 & $0.0000 E+00$ & $1.0000 E-20$ & $2.0000 E-20$ & $1.0000 E-20$ & $1.0000 E-20$ \\
0.04 & $0.0000 E+00$ & $0.0000 E+00$ & $3.0000 E-20$ & $2.0000 E-20$ & $2.0000 E-20$ \\
0.05 & $1.0000 E-20$ & $0.0000 E+00$ & $1.0000 E-20$ & $0.0000 E+00$ & $1.0000 E-20$ \\
\hline
\end{tabular}

We obtain the first few Adomian polynomials for $\Phi(u)=u^{m} u_{x}$ as

$$
\begin{aligned}
A_{0}= & u_{0}^{m}\left(u_{0}\right)_{x^{\prime}} \\
A_{1}= & m u_{0}^{m-1} u_{1}\left(u_{0}\right)_{x}+u_{0}^{m}\left(u_{1}\right)_{x} \\
A_{2}= & m u_{0}^{m-1} u_{2}\left(u_{0}\right)_{x}+m u_{0}^{m-1} u_{1}\left(u_{1}\right)_{x}+u_{0}^{m}\left(u_{2}\right)_{x}+\frac{m}{2}(m-1) u_{0}^{m-2} u_{1}^{2}\left(u_{0}\right)_{x} \\
A_{3}= & m u_{0}^{m-1} u_{3}\left(u_{0}\right)_{x}+m(m-1) u_{0}^{m-2} u_{1} u_{2}\left(u_{0}\right)_{x}+\frac{m}{2}(m-1) u_{0}^{m-2} u_{1}^{3}\left(u_{0}\right)_{x} \\
& +m u_{0}^{m-1} u_{2}\left(u_{1}\right)_{x}+m u_{0}^{m-1} u_{1}\left(u_{2}\right)_{x}+u_{0}^{m}\left(u_{3}\right)_{x^{\prime}} \\
\quad \vdots &
\end{aligned}
$$

In Section 3, we provide some examples and demonstrate the absolute errors $\mid u(x, t)-$ $\phi_{n}(x, t) \mid$ in Tables 5-6, where $u(x, t)$ is the particular analytical solution and $\phi_{n}(x, t)$ is the partial sum:

$$
\phi_{n}(x, t)=\sum_{k=0}^{n} u_{k}(x, t), \quad n \geq 0
$$

Equations of the form (1.10) admit traveling-wave solutions $u=u(k x+\lambda t)$ where $k$ and $\lambda$ are constants [48]. 
Table 5: Numerical results for $\left|u(x, t)-\phi_{50}(x, t)\right|$ where $u(x, t)=1 /(x-t)$ for (3.33).

\begin{tabular}{lccccc}
\hline$t_{i} \mid x_{i}$ & 0.5 & 0.6 & 0.65 & 0.7 & 0.8 \\
\hline 0.11 & $0.0000 E+00$ & $0.0000 E+00$ & $0.0000 E+00$ & $0.0000 E+00$ & $0.0000 E+00$ \\
0.2 & $0.0000 E+00$ & $1.3322 E-15$ & $1.3322 E-15$ & $0.0000 E+00$ & $0.0000 E+00$ \\
0.3 & $4.0413 E-11$ & $2.6645 E-15$ & $0.0000 E+00$ & $0.0000 E+00$ & $0.0000 E+00$ \\
0.4 & $1.4272 E-4$ & $7.8416 E-9$ & $1.1465 E-10$ & $2.3510 E-12$ & $2.2204 E-15$ \\
0.45 & $0.1030 E+00$ & $3.7754 E-6$ & $5.1752 E-8$ & $1.0180 E-9$ & $9.1571 E-13$ \\
\hline
\end{tabular}

Table 6: Numerical results for $\left|u(x, t)-\phi_{6}(x, t)\right|$ where $u(x, t)=(2 x-3 t)^{-3 / 4}$ for (3.38).

\begin{tabular}{lccccc}
\hline$t_{i} \mid x_{i}$ & 1 & 2.5 & 4.8 & 8 & 10.8 \\
\hline 0.01 & $5.1070 E-14$ & $0.0000 E+00$ & $0.0000 E+00$ & $0.0000 E+00$ & $0.0000 E+00$ \\
0.1 & $5.8857 E-7$ & $4.4009 E-10$ & $2.7246 E-12$ & $5.1348 E-14$ & $5.0238 E-15$ \\
0.2 & $9.0812 E-5$ & $6.0039 E-8$ & $3.5999 E-10$ & $6.6973 E-12$ & $6.4802 E-13$ \\
0.3 & $1.9539 E-3$ & $1.0982 E-6$ & $6.3556 E-9$ & $1.1663 E-10$ & $1.1227 E-11$ \\
0.45 & $0.0548 E+00$ & $2.0984 E-5$ & $1.1431 E-7$ & $2.0519 E-9$ & $1.9595 E-10$ \\
\hline
\end{tabular}

\section{Applications of ADM}

Example 3.1. We first consider (1.3) with initial data $u(0)=N_{0} / K$. We proceed as in Section 2.1. We take $f(u)=u^{2}$ and $\gamma=N_{0} / K$. Adomian polynomials can be derived as follows:

$$
\begin{aligned}
f(u)= & u^{2}=\sum_{n=0}^{\infty} A_{n}=\left(u_{0}+u_{1}+u_{2}+\cdots\right)^{2} \\
= & \left(u_{0}^{2}\right)+\left(2 u_{0} u_{1}\right)+\left(2 u_{0} u_{2}+u_{1}^{2}\right)+\left(2 u_{0} u_{3}+2 u_{1} u_{2}\right) \\
& +\left(2 u_{0} u_{4}+2 u_{1} u_{3}+u_{2}^{2}\right)+\left(2 u_{0} u_{5}+2 u_{1} u_{4}+2 u_{2} u_{3}\right)+\cdots
\end{aligned}
$$

Therefore, we get the following Adomian polynomials [14]:

$$
\begin{aligned}
& A_{0}=u_{0}^{2}, \\
& A_{1}=2 u_{0} u_{1}, \\
& A_{2}=2 u_{0} u_{2}+u_{1}^{2}, \\
& A_{3}=2 u_{0} u_{3}+2 u_{1} u_{2}, \\
& A_{4}=2 u_{0} u_{4}+2 u_{1} u_{3}+u_{2}^{2}, \\
& A_{5}=2 u_{0} u_{5}+2 u_{1} u_{4}+2 u_{2} u_{3},
\end{aligned}
$$


For numerical purposes we take $N_{0}=2$ and $K=1$. Therefore,

$$
\begin{aligned}
& u_{0}=u(0)=N_{0} / K=2, \\
& u_{1}=L^{-1} u_{0}-L^{-1} A_{0}=-2 \tau, \\
& u_{2}=L^{-1} u_{1}-L^{-1} A_{1}=3 \tau^{2}, \\
& u_{3}=L^{-1} u_{2}-L^{-1} A_{2}=-13 / 3 \tau^{3}, \\
& u_{4}=L^{-1} u_{3}-L^{-1} A_{3}=25 / 4 \tau^{4}, \\
& u_{5}=L^{-1} u_{4}-L^{-1} A_{4}=-541 / 60 \tau^{5},
\end{aligned}
$$

and so on, in this manner the rest of the terms of the decomposition series have been calculated using Mathcad7. Substituting these terms into (2.2), we obtain

$$
\begin{aligned}
u(\tau) & =u_{0}(\tau)+u_{1}(\tau)+u_{2}(\tau)+u_{3}(\tau)+u_{4}(\tau)+u_{5}(\tau)+\cdots \\
& =2-2 \tau+3 \tau^{2}-13 / 3 \tau^{3}+25 / 4 \tau^{4}-541 / 60 \tau^{5}+\cdots
\end{aligned}
$$

which gives the analytical solution obtained in (1.4) in the closed form, with $N_{0}=2, K=1$. We let $\phi_{n}$ be the $n$th partial sums of the series in (2.2), that is,

$$
\phi_{n}=\sum_{k=0}^{n} u_{k}, \quad n \geq 0,
$$

and compare the analytical solution with (3.4) in Figure 1.

Example 3.2. We now consider the initial value problem given by (1.7) with initial data $u(0)=$ $\delta=1.3, v(0)=\beta=0.6$. We proceed as in Section 2.2. We take $\alpha=1, f(u, v)=g(u, v)=u v$. Therefore, from (2.13) we obtain $B_{n}=C_{n}, n=0,1,2, \ldots$, and Adomian polynomials can be derived as follows:

$$
\begin{aligned}
f(u, v) & =g(u, v) \\
& =u v \\
& =\sum_{n=0}^{\infty} B_{n} \\
& =\left(\sum_{n=0}^{\infty} u_{n}\right)\left(\sum_{n=0}^{\infty} v_{n}\right) \\
& =\sum_{n=0}^{\infty}\left(\sum_{k=0}^{n} u_{k} v_{n-k}\right) .
\end{aligned}
$$




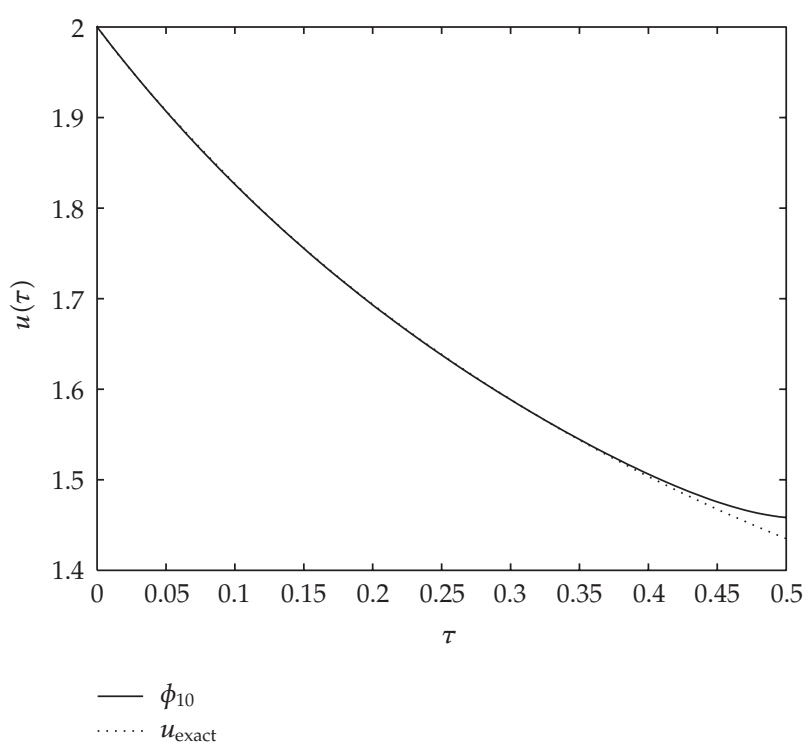

Figure 1: Solution to the logistic growth model $((1.3)$ with $u(0)=2)$ in a population.

Hence, we get the following Adomian polynomials:

$$
B_{n}=C_{n}=\sum_{k=0}^{n} u_{k} v_{n-k}, \quad n=0,1,2, \ldots
$$

From this equality, we have

$$
\begin{aligned}
& B_{0}=C_{0}=u_{0} v_{0}, \\
& B_{1}=C_{1}=u_{0} v_{1}+u_{1} v_{0}, \\
& B_{2}=C_{2}=u_{0} v_{2}+u_{1} v_{1}+u_{2} v_{0}, \\
& B_{3}=C_{3}=u_{0} v_{3}+u_{1} v_{2}+u_{2} v_{1}+u_{3} v_{0},
\end{aligned}
$$

Let us now compute the $u_{k}$ and $v_{k}$ from (2.17):

$$
\begin{aligned}
& u_{0}=u(0)=\delta=1.3, \\
& v_{0}=v(0)=\beta=0.6, \\
& u_{1}=L^{-1} u_{0}-L^{-1} B_{0}=0.52 \tau, \\
& v_{1}=L^{-1} C_{0}-L^{-1} v_{0}=0.18 \tau,
\end{aligned}
$$




$$
\begin{aligned}
& u_{2}=L^{-1} u_{1}-L^{-1} B_{1}=-0.013 \tau^{2}, \\
& v_{2}=L^{-1} C_{1}-L^{-1} v_{1}=0.1830 \tau^{2}, \\
& u_{3}=L^{-1} u_{2}-L^{-1} B_{2}=-0.1122 \tau^{3}, \\
& v_{3}=L^{-1} C_{2}-L^{-1} v_{2}=0.0469 \tau^{3}, \\
& u_{4}=L^{-1} u_{3}-L^{-1} B_{3}=-0.0497 \tau^{4}, \\
& v_{4}=L^{-1} C_{3}-L^{-1} v_{3}=0.0099 \tau^{4},
\end{aligned}
$$

and so on, in this manner the rest of the terms of the decomposition series have been calculated using Mathcad7. Substituting these terms into (2.12), we obtain the following approximate solutions to the initial value problem given by (1.7) with initial data $u(0)=\delta=$ $1.3, v(0)=\beta=0.6$ :

$$
\begin{aligned}
u(\tau) & =u_{0}(\tau)+u_{1}(\tau)+u_{2}(\tau)+u_{3}(\tau)+u_{4}(\tau)+\cdots \\
& =1.3+0.52 \tau-0.013 \tau^{2}-0.1122 \tau^{3}-0.0497 \tau^{4}-\cdots \\
v(\tau) & =v_{0}(\tau)+v_{1}(\tau)+v_{2}(\tau)+v_{3}(\tau)+v_{4}(\tau)+\cdots \\
& =0.6+0.18 \tau+0.1830 \tau^{2}+0.0469 \tau^{3}+0.0099 \tau^{4}+\cdots
\end{aligned}
$$

Example 3.3. If we take $\epsilon=1$ and $m=1$ in the (1.8), we obtain the linear heat equation, namely,

$$
u_{t}=u_{x x}+u
$$

We impose the initial condition

$$
u(x, 0)=\cos (\pi x),
$$

and boundary conditions

$$
u(0, t)=e^{\left(1-\pi^{2}\right) t}, \quad u_{x}(0, t)=0 .
$$

To obtain the solution in $t$ direction, we use the recursive relation (2.22) by simply taking $u_{0}=\cos (\pi x)$. In this case the Adomian Polynomials are $A_{0}=u_{0}, A_{1}=u_{1}, A_{2}=u_{2}$, and so on. Therefore, we have

$$
u_{1}=\left(1-\pi^{2}\right) t \cos (\pi x), \quad u_{2}=\frac{1}{2 !}\left(1-\pi^{2}\right)^{2} t^{2} \cos (\pi x), \quad u_{3}=\frac{1}{3 !}\left(1-\pi^{2}\right)^{3} t^{3} \cos (\pi x),
$$


and so on, in this manner the rest of the components of the series (2.21) have been calculated using Mathcad7. Putting these individual terms in (2.21) one gets the analytical solution:

$$
\begin{aligned}
u(x, t) & =\cos (\pi x)+\left(1-\pi^{2}\right) t \cos (\pi x)+\frac{1}{2 !}\left(1-\pi^{2}\right) t^{2} \cos (\pi x)+\frac{1}{3 !}\left(1-\pi^{2}\right)^{3} t^{3} \cos (\pi x)+\cdots \\
& =e^{\left(1-\pi^{2}\right) t} \cos (\pi x)
\end{aligned}
$$

which can be verified through substitution.

Similarly, to obtain the solution in $x$ direction, we use the recursive relation (2.23) by taking $u_{0}=e^{\left(1-\pi^{2}\right) t}$, where the $A_{n}$ are the same as above. We therefore have

$$
u_{1}=-\frac{(\pi x)^{2}}{2 !} e^{\left(1-\pi^{2}\right) t}, \quad u_{2}=\frac{(\pi x)^{4}}{4 !} e^{\left(1-\pi^{2}\right) t}, \quad u_{3}=-\frac{(\pi x)^{6}}{6 !} e^{\left(1-\pi^{2}\right) t}
$$

and so on, in this manner the rest of the components of the series (2.21) have been calculated. From the decomposition series (2.21), we again obtain the analytical solution:

$$
\begin{aligned}
u(x, t) & =e^{\left(1-\pi^{2}\right) t}-\frac{(\pi x)^{2}}{2 !} e^{\left(1-\pi^{2}\right) t}+\frac{(\pi x)^{4}}{4 !} e^{\left(1-\pi^{2}\right) t}-\frac{(\pi x)^{6}}{6 !} e^{\left(1-\pi^{2}\right) t}+\cdots \\
& =e^{\left(1-\pi^{2}\right) t} \cos (\pi x) .
\end{aligned}
$$

Example 3.4. In the second example, we consider the nonlinear heat equation (1.8) with $\epsilon=-2$ and $m=3$, that is,

$$
u_{t}=u_{x x}-2 u^{3}
$$

In [46] the authors solve (3.19) using antireduction method, and give the solution by means of ansatz $\left(\varphi_{i}, i=1,2\right)$ as follows:

$$
u(x, t)=\left(\varphi_{1}(t)+2 \varphi_{2}(t) x\right)\left(1+\varphi_{1}(t) x+\varphi_{2}(t) x^{2}\right)^{-1},
$$

where $\varphi_{1}$ and $\varphi_{2}$ satisfy the ordinary differential equations:

$$
\varphi_{1}^{\prime}=-6 \varphi_{1} \varphi_{2}, \quad \varphi_{2}^{\prime}=-6 \varphi_{2}^{2}
$$

We impose $\varphi_{1}(0)=\varphi_{2}(0)=1$, and solve the above ordinary differential equations, and obtain

$$
\varphi_{1}(t)=\varphi_{2}(t)=\frac{1}{6 t+1}
$$

Therefore, we have the partial analytical solution of (3.19) as

$$
u(x, t)=\frac{1+2 x}{x^{2}+x+6 t+1} .
$$


We now solve (3.19) using ADM with the initial condition:

$$
u(x, 0)=\frac{1+2 x}{x^{2}+x+1}
$$

and the boundary conditions:

$$
u(0, t)=\frac{1}{6 t+1}, \quad u_{x}(0, t)=\frac{12 t+1}{(6 t+1)^{2}}
$$

For the solution of this equation in the $t$ direction, we use the recursive relation given by (2.22) to obtain the terms of the decomposition series (2.25). In this case the Adomian Polynomials are $A_{0}=-2 u_{0}^{3}, A_{1}=-6 u_{0}^{2} u_{1}, A_{2}=-6\left(u_{0} u_{1}^{2}+u_{0}^{2} u_{2}\right)$, and so on. Therefore, we obtain

$$
\begin{aligned}
& u_{0}=\frac{1+2 x}{x^{2}+x+1} \\
& u_{1}=L_{t}^{-1}\left(L_{x}\left(u_{0}\right)\right)-2 L_{t}^{-1}\left(u_{0}^{3}\right)=\frac{-6(1+2 x)}{\left(x^{2}+x+1\right)^{2}} t \\
& u_{2}=L_{t}^{-1}\left(L_{x}\left(u_{1}\right)\right)-6 L_{t}^{-1}\left(u_{0}^{2} u_{1}\right)=\frac{36(1+2 x)}{\left(x^{2}+x+1\right)^{3}} t^{2}, \\
& u_{3}=L_{t}^{-1}\left(L_{x}\left(u_{2}\right)\right)-6 L_{t}^{-1}\left(u_{0}^{2} u_{2}+u_{1}^{2} u_{0}\right)=\frac{-216(1+2 x)}{\left(x^{2}+x+1\right)^{4}} t^{3},
\end{aligned}
$$

and so on, in this manner the rest of the terms of the decomposition series have been calculated using Mathcad7. Substituting (3.26) into the decomposition series (2.25), we obtain

$$
\begin{aligned}
u(x, t)= & u_{0}(x, t)+u_{1}(x, t)+u_{2}(x, t)+u_{3}(x, t) \cdots \\
= & \frac{1+2 x}{x^{2}+x+1}-\frac{6(1+2 x)}{\left(x^{2}+x+1\right)^{2}} t \\
& +\frac{36(1+2 x)}{\left(x^{2}+x+1\right)^{3}} t^{2}-\frac{216(1+2 x)}{\left(x^{2}+x+1\right)^{4}} t^{3} \cdots
\end{aligned}
$$

which gives the analytical solution obtained in (3.23) in the closed form. This result can be verified through substitution. 
On the other hand, to obtain the solution in the $x$ direction, we use the recursive relation given by (2.23) to determine the individual terms of the series (2.25):

$$
\begin{aligned}
u_{0}= & \frac{1}{6 t+1}+x\left(\frac{12 t+1}{(6 t+1)^{2}}\right) \\
u_{1}= & L_{x}{ }^{-1}\left(L_{t}\left(u_{0}\right)\right)+2 L_{x}{ }^{-1}\left(u_{0}^{3}\right) \\
= & x^{2}\left(\frac{-18 t-2}{(6 t+1)^{3}}\right)+x^{3}\left(\frac{-72 t^{2}+1}{(6 t+1)^{4}}\right) \\
& +x^{4}\left(\frac{180 t^{2}+30 t+1}{(6 t+1)^{5}}\right)+x^{5}\left(\frac{432 t^{3}-108 t^{2}-36 t-2}{(6 t+1)^{6}}\right),
\end{aligned}
$$

and so on. In this manner the rest of the terms of the decomposition series (2.25) have been calculated. Substituting (3.28) into (2.25) gives

$$
\begin{aligned}
u(x, t)= & u_{0}(x, t)+u_{1}(x, t)+\cdots \\
= & \frac{1}{6 t+1}+x\left(\frac{12 t+1}{(6 t+1)^{2}}\right)+x^{2}\left(\frac{-18 t-2}{(6 t+1)^{3}}\right) \\
& +x^{3}\left(\frac{-72 t^{2}+1}{(6 t+1)^{4}}\right)+x^{4}\left(\frac{180 t^{2}+30 t+1}{(6 t+1)^{5}}\right) \\
& +x^{5}\left(\frac{432 t^{3}-108 t^{2}-36 t-2}{(6 t+1)^{6}}\right) \cdots,
\end{aligned}
$$

which again gives the analytical solution given by (3.23) in the closed form.

Example 3.5. Let us take $m=1$ in (1.10). We obtain

$$
\frac{\partial u}{\partial t}=\frac{\partial}{\partial x}\left(u \frac{\partial u}{\partial x}\right)
$$

We impose the initial condition

$$
u(x, 0)=x
$$

To obtain the solution, we use the recursive relation (2.30) by taking $u_{0}=x$. In this case the first Adomian Polynomial is $A_{0}=x$. Therefore, we have $u_{1}=t$ and $A_{1}=t$. Finally, 
$u_{2}=0$ which follows that $u_{n}(x, t)=0$ for $n \geq 2$. Putting these individual terms in (2.29), one gets the analytical solution

$$
u(x, t)=x+t
$$

which can be verified through substitution.

Example 3.6. When $m=-1$, (1.10) becomes

$$
\frac{\partial u}{\partial t}=\frac{\partial}{\partial x}\left(\frac{1}{u} \frac{\partial u}{\partial x}\right)
$$

In [48] the authors give a particular analytical solution to (3.33) as follows:

$$
u(x, t)=\left(c_{1} x-c_{1}^{2} t+c_{2}\right)^{-1}
$$

where $c_{1}$ and $c_{2}$ are arbitrary constants. We take $c_{1}=1$ and $c_{2}=0$ for simplicity. Therefore, with these choices of $c_{1}$ and $c_{2}$ their solution becomes

$$
u(x, t)=\frac{1}{x-t} .
$$

We now solve (3.33) using ADM with the initial condition:

$$
u(x, 0)=\frac{1}{x}
$$

For the solution of this equation, we use the recursive relation given by (2.30) to obtain the terms of the decomposition series (2.29). In this case $u_{0}=1 / x, A_{0}=-1 / x, u_{1}=t / x^{2}$, $A_{1}=-t / x^{2}, u_{2}=t^{2} / x^{3}, A_{2}=-t^{2} / x^{3}, u_{3}=t^{3} / x^{4}$, and so on. In this manner the rest of the terms of the decomposition series have been calculated using Mathcad7. Substituting these individual terms in (2.29), we obtain

$$
\begin{aligned}
u(x, t) & =u_{0}(x, t)+u_{1}(x, t)+u_{2}(x, t)+u_{3}(x, t)+\cdots \\
& =\frac{1}{x}+\frac{t}{x^{2}}+\frac{t^{2}}{x^{3}}+\frac{t^{3}}{x^{4}}+\cdots
\end{aligned}
$$

which gives the analytical solution obtained in (3.35) in the closed form. This result can be verified through substitution.

Example 3.7. If $m=-4 / 3,(1.10)$ reads

$$
\frac{\partial u}{\partial t}=\frac{\partial}{\partial x}\left(u^{-4 / 3} \frac{\partial u}{\partial x}\right)
$$


In [48], a particular analytical solution to (3.38) is given as follows:

$$
u(x, t)=\left(2 c_{1} x-3 c_{1}^{2} t+c_{2}\right)^{-3 / 4}
$$

where $c_{1}$ and $c_{2}$ are arbitrary constants, and we take $c_{1}=1$ and $c_{2}=0$ for simplicity. Therefore, one has

$$
u(x, t)=(2 x-3 t)^{-3 / 4}
$$

We now solve (3.38) using ADM with the initial condition:

$$
u(x, 0)=(2 x)^{-3 / 4}
$$

We again use the recursive relation given by (2.30) to obtain the terms of the decomposition series (2.29). In this case $u_{0}=(2 x)^{-3 / 4}, A_{0}=-3 \times 2^{-7 / 4} x^{-3 / 4}, u_{1}=9 \times 2^{-15 / 4} x^{-7 / 4} t, A_{1}=-27 \times$ $2^{-19 / 4} x^{-7 / 4} t, u_{2}=189 \times 2^{-31 / 4} x^{-11 / 4} t^{2}, A_{2}=-567 \times 2^{-35 / 4} x^{-11 / 4} t^{2}, u_{3}=2079 \times 2^{-43 / 4} x^{-15 / 4} t^{3}$, and so on. In this manner the rest of the terms of the decomposition series have been calculated using Mathcad7. Substituting these individual terms in (2.29), one obtains

$$
\begin{aligned}
u(x, t)= & u_{0}(x, t)+u_{1}(x, t)+u_{2}(x, t)+u_{3}(x, t)+\cdots \\
= & (2 x)^{-3 / 4}+9 \times 2^{-15 / 4} x^{-7 / 4} t+189 \times 2^{-31 / 4} x^{-11 / 4} t^{2} \\
& +2079 \times 2^{-43 / 4} x^{-15 / 4} t^{3}+\cdots
\end{aligned}
$$

which gives the analytical solution obtained in (3.40) in the closed form. This result can also be verified through substitution.

\section{The Idea of Homotopy Perturbation Method}

The basic idea of the homotopy perturbation method (HPM) can be illustrated as follows [19]: we consider the nonlinear differential equation:

$$
A(u)-f(\mathbf{r})=0, \quad \mathbf{r} \in \Omega,
$$

with boundary conditions:

$$
B(u, \partial u / \partial n)=0, \quad \mathbf{r} \in \Gamma
$$

where $A$ is a general differential operator, $B$ is a boundary operator, $f(\mathbf{r})$ is a known analytic function, and $\Gamma$ is the boundary of the domain $\Omega$.

In general, one divides the operator $A$ into two parts $L$ and $N$, where $L$ is linear, while $N$ is nonlinear. Therefore, (4.1) is written as follows:

$$
L(u)+N(u)-f(\mathbf{r})=0 .
$$


By the homotopy technique $[19,51]$, one constructs a homotopy $v(\mathbf{r}, p): \Omega \times[0,1] \rightarrow \mathbf{R}$ which satisfies

$$
H(v, p)=(1-p)\left[L(v)-L\left(u_{0}\right)\right]+p[A(v)-f(\mathbf{r})]=0, \quad p \in[0,1], \mathbf{r} \in \Omega,
$$

or

$$
H(v, p)=L(v)-L\left(u_{0}\right)+p L\left(u_{0}\right)+p[N(v)-f(\mathbf{r})]=0,
$$

where $p \in[0,1]$ is an embedding parameter, $u_{0}$ is an initial approximation of $(4.1)$, which satisfies the boundary conditions. It is clear that

$$
\begin{aligned}
& H(v, 0)=L(v)-L\left(u_{0}\right)=0, \\
& H(v, 1)=A(v)-f(\mathbf{r})=0,
\end{aligned}
$$

the changing process of $p$ from zero to unity is just that of $v(\mathbf{r}, p)$ from $u_{0}(\mathbf{r})$ to $u(\mathbf{r})$.

According to the HPM, we can first use the embedding parameter $p$ as a "small parameter", and assume that the solution of (4.4) and (4.5) can be written as a power series in $p$ :

$$
v=v_{0}+p v_{1}+p^{2} v_{2}+\cdots
$$

Setting $p=1$ results in the approximate solution of (4.1):

$$
u=\lim _{p \rightarrow 1} v=v_{0}+v_{1}+v_{2}+\cdots
$$

The combination of the perturbation method and the homotopy method is called the homotopy perturbation method, which has eliminated limitations of the traditional perturbation methods.

The series (4.8) is convergent for most cases, however, the convergent rate depends on the nonlinear operator $A(v)$ (the following opinions are suggested by He [19]).

(1) The second derivative of $N(v)$ with respect to $v$ must be small because the parameter may be relatively large, that is, $p \rightarrow 1$.

(2) The norm of $L^{-1} \partial N / \partial v$ must be smaller than one so that the series converges. 


\section{Applications of HPM}

Example 5.1. We now solve (1.3) using HPM with the initial condition $u(0)=2$, as chosen in Example 3.1. We rewrite (1.3) in the form [52]

$$
\begin{gathered}
\frac{d u}{d \tau}=p u(1-u), \\
u(0)=2,
\end{gathered}
$$

where $p \in[0,1]$ is an embedding parameter. As in He's HPM, it is clear that when $p=0,(5.1)$ becomes a linear equation; when $p=1$, it becomes the original nonlinear one. We consider the imbedding parameter $p$ as a "small parameter". We assume the solution of (5.1) is expressed as a power series given in (4.7). Substituting (4.7) into (5.1), and equating coefficients of like $p$, we obtain the following differential equations:

$$
\begin{aligned}
& p^{0}:\left\{v_{0}^{\prime}=0, \quad v_{0}(0)=2,\right. \\
& p^{1}:\left\{v_{1}^{\prime}=v_{0}-v_{0}^{2}, \quad v_{1}(0)=0,\right. \\
& p^{2}:\left\{v_{2}^{\prime}=v_{1}-2 v_{0} v_{1}, \quad v_{2}(0)=0,\right. \\
& p^{3}:\left\{v_{3}^{\prime}=v_{2}-v_{1}^{2}-2 v_{0} v_{2}, \quad v_{3}(0)=0,\right. \\
& p^{4}:\left\{v_{4}^{\prime}=v_{3}-2 v_{0} v_{3}-2 v_{1} v_{2}, \quad v_{4}(0)=0,\right. \\
& p^{5}:\left\{v_{5}^{\prime}=v_{4}-v_{2}^{2}-2 v_{1} v_{3}-2 v_{0} v_{4}, \quad v_{5}(0)=0,\right.
\end{aligned}
$$

where "primes" denote differentiation with respect to $\tau$. Thus, solving the equations above yields

$$
\begin{aligned}
& v_{0}=2, \\
& v_{1}=-2 \tau, \\
& v_{2}=3 \tau^{2}, \\
& v_{3}=-13 / 3 \tau^{3}, \\
& v_{4}=25 / 4 \tau^{4}, \\
& v_{5}=-541 / 60 \tau^{5},
\end{aligned}
$$


Substituting these in (4.7) gives

$$
v=2-2 p \tau+3 p^{2} \tau^{2}-13 / 3 p^{3} \tau^{3}+25 / 4 p^{4} \tau^{4}-541 / 60 p^{5} \tau^{5}+\cdots
$$

Hence, by (4.8) one has

$$
u=2-2 \tau+3 \tau^{2}-13 / 3 \tau^{3}+25 / 4 \tau^{4}-541 / 60 \tau^{5}+\cdots,
$$

which is exactly the same solution obtained in (3.4). Also, the solution in (5.5) is equal to

$$
u=\frac{2}{2-e^{-\tau}}
$$

in the closed form which is exactly the same as in (1.4) with $K=1, N_{0}=2$ (see Example 3.1).

Example 5.2. We now solve (1.7) using HPM with $\alpha=1, u(0)=1.3, v(0)=0.6$ as taken in Example 3.2. We rewrite (1.7) in the form [52]

$$
\frac{d u}{d \tau}=p u(1-v), \quad \frac{d v}{d \tau}=p v(u-1), \quad u(0)=1.3, \quad v(0)=0.6
$$

where $p \in[0,1]$ is an embedding parameter. As in He's HPM, it is clear that when $p=0$, (5.7) becomes a linear system; when $p=1$, it becomes the original nonlinear one. We consider the imbedding parameter $p$ as a "small parameter". We assume the solutions of $(5.7),(u, v)$ are expressed as power series:

$$
\begin{aligned}
& u=u_{0}+p u_{1}+p^{2} u_{2}+\cdots \\
& v=v_{0}+p v_{1}+p^{2} v_{2}+\cdots
\end{aligned}
$$

respectively. Substituting (5.8) and (5.9) into the system (5.7), and equating coefficients of like $p$, we obtain the following systems of differential equations:

$$
\begin{aligned}
& p^{0}:\left\{\begin{array}{l}
u_{0}^{\prime}=0 \\
v_{0}^{\prime}=0 \\
u_{0}(0)=1.3, \quad v_{0}(0)=0.6,
\end{array}\right. \\
& p^{1}:\left\{\begin{array}{l}
u_{1}^{\prime}=u_{0}\left(1-v_{0}\right) \\
v_{1}^{\prime}=v_{0}\left(u_{0}-1\right) \\
u_{1}(0)=0, \quad v_{1}(0)=0,
\end{array}\right.
\end{aligned}
$$


Mathematical Problems in Engineering

$$
\begin{gathered}
p^{2}:\left\{\begin{array}{l}
u_{2}^{\prime}=u_{1}-\left(u_{0} v_{1}+u_{1} v_{0}\right) \\
v_{2}^{\prime}=u_{0} v_{1}+u_{1} v_{0}-v_{1} \\
u_{2}(0)=0, \quad v_{2}(0)=0,
\end{array}\right. \\
p^{3}:\left\{\begin{array}{l}
u_{3}^{\prime}=u_{2}-\left(u_{0} v_{2}+u_{1} v_{1}+u_{2} v_{0}\right) \\
v_{3}^{\prime}=u_{0} v_{2}+u_{1} v_{1}+u_{2} v_{0}-v_{2} \\
u_{3}(0)=0, \quad v_{3}(0)=0,
\end{array}\right. \\
p^{4}:\left\{\begin{array}{l}
u_{4}^{\prime}=u_{3}-\left(u_{0} v_{3}+u_{1} v_{2}+u_{2} v_{1}+u_{3} v_{0}\right) \\
v_{4}^{\prime}=u_{0} v_{3}+u_{1} v_{2}+u_{2} v_{1}+u_{3} v_{0}-v_{3} \\
u_{4}(0)=0, \quad v_{4}(0)=0,
\end{array}\right. \\
\vdots \quad
\end{gathered}
$$

where "primes" denote differentiation with respect to $\tau$. Thus, solving the above systems of equations yields

$$
\begin{aligned}
& u_{0}=1.3, \quad v_{0}=0.6, \\
& u_{1}=0.52 \tau, \quad v_{1}=0.18 \tau, \\
& u_{2}=-0.013 \tau^{2}, \quad v_{2}=0.183 \tau^{2}, \\
& u_{3}=-0.1122 \tau^{3}, \quad v_{3}=0.0469 \tau^{3}, \\
& u_{4}=-0.0497 \tau^{4}, \quad v_{4}=0.0099 \tau^{4},
\end{aligned}
$$

Substituting these $u_{n}, v_{n}, n \geq 0$ into (5.8), and (5.9), respectively, we have

$$
\begin{aligned}
& u=1.3+0.52 p \tau-0.013 p^{2} \tau^{2}-0.1122 p^{3} \tau^{3}-0.0497 p^{4} \tau^{4}-\cdots, \\
& v=0.6+0.18 p \tau+0.183 p^{2} \tau^{2}+0.0469 p^{3} \tau^{3}+0.0099 p^{4} \tau^{4}+\cdots
\end{aligned}
$$

Letting $p \rightarrow 1$ one obtains

$$
\begin{aligned}
& u=1.3+0.52 \tau-0.013 \tau^{2}-0.1122 \tau^{3}-0.0497 \tau^{4}-\cdots, \\
& v=0.6+0.18 \tau+0.183 \tau^{2}+0.0469 \tau^{3}+0.0099 \tau^{4}+\cdots,
\end{aligned}
$$

which are exactly the same solutions obtained in (3.10) and (3.11), respectively. 


\section{The Modified HPM for the Porous Media Equation}

In this section we outline the modified HPM studied by Chun et al. [35]. In this work, they have solved the porous media equation

$$
u_{t}=\left(u^{m} u_{x}\right)_{x}
$$

using modified HPM with initial condition:

$$
u(x, 0)=f(x)
$$

Here $u=u(x, t)$. Equation (6.1) is the same equation we have solved in Section 2.4 using ADM, and we have provided some numerical examples for it in Examples 3.5-3.7. In their work they construct the following homotopy with $L(u)=u_{t}$ and $N(u)=-\left(u^{m} u_{x}\right)_{x}$,

$$
L(v)-L\left(u_{0}\right)+p L\left(u_{0}\right)+p N(v)=0 .
$$

To deal with the nonlinear term, they employ He polynomials considered in $[33,34]$ which is given by

$$
N(u)=N\left(v_{0}\right)+N\left(v_{0}, v_{1}\right) p+N\left(v_{0}, v_{1}, v_{2}\right) p^{2}+\cdots+N\left(v_{0}, v_{1}, \ldots, v_{n}\right) p^{n}+\cdots,
$$

where

$$
N\left(v_{0}, v_{1}, \ldots, v_{n}\right)=\frac{1}{n !} \frac{\partial^{n}}{\partial p^{n}}\left[N\left(\sum_{k=0}^{n} p^{k} v_{k}\right)\right]_{p=0}, \quad n=1,2, \ldots
$$

Substituting (6.4) into (6.3), and equating coefficients of like $p$, one obtains

$$
\begin{aligned}
& p^{0}: L\left(v_{0}\right)-L\left(u_{0}\right)=0, \\
& p^{1}: L\left(v_{1}\right)+L\left(u_{0}\right)+N\left(v_{0}\right)=0, \\
& p^{2}: L\left(v_{2}\right)+N\left(v_{0}, v_{1}\right)=0, \\
& p^{3}: L\left(v_{3}\right)+N\left(v_{0}, v_{1}, v_{2}\right)=0, \\
& \quad \vdots \\
& p^{n+1}: L\left(v_{n+1}\right)+N\left(v_{0}, v_{1}, \ldots, v_{n}\right)=0,
\end{aligned}
$$


and so on, which forms the basis of a complete determination of the components $v_{0}, v_{1}, \ldots, v_{n}, \ldots$. They let $u_{0}(x, t)=0$ for simplicity. Therefore, they obtain the following linear equations for these components:

$$
\begin{gathered}
\left(v_{0}\right)_{t}=0, \quad v_{0}(x, 0)=f(x), \\
\left(v_{1}\right)_{t}-\left(v_{0}^{m} v_{0_{x}}\right)_{x}=0, \quad v_{1}(x, 0)=0, \\
\left(v_{2}\right)_{t}+\frac{\partial}{\partial p}\left[N\left(\sum_{k=0}^{1} p^{k} v_{k}\right)\right]_{p=0}=0, \quad v_{2}(x, 0)=0, \\
\left(v_{3}\right)_{t}+\frac{1}{2 !} \frac{\partial^{2}}{\partial p^{2}}\left[N\left(\sum_{k=0}^{2} p^{k} v_{k}\right)\right]_{p=0}=0, \quad v_{3}(x, 0)=0,
\end{gathered}
$$

and so on.

Example 6.1. In this example we solve the initial value problem given by (3.30)-(3.31) using modified HPM. We simply take $m=1$ and $f(x)=x$ in (6.1) and (6.2), respectively, and use (6.7) $-(6.10)$ to obtain the components $v_{0}, v_{1}, \ldots, v_{n}, \ldots$. From (6.7)-(6.9), we easily obtain $v_{0}(x, t)=x, v_{1}(x, t)=t$, and $v_{2}(x, t)=0$. Therefore, one gets $v_{n}(x, t)=0, n \geq 2$, which results in

$$
u(x, t)=x+t
$$

This is exactly the same solution we have obtained in (3.32).

Example 6.2. We now solve the initial value problem given by (3.33) and (3.36) using the same method. We now take $m=-1$ and $f(x)=1 / x$ in (6.1) and (6.2), respectively, and use (6.7)-(6.10). After somewhat tedious computations we obtain $v_{0}(x, t)=1 / x, v_{1}(x, t)=t / x^{2}$, $v_{2}(x, t)=t^{2} / x^{3}, v_{3}(x, t)=t^{3} / x^{4}$, and so on. Therefore, one gets the solution to this problem as follows:

$$
u(x, t)=1 / x+t / x^{2}+t^{2} / x^{3}+t^{3} / x^{4}+\cdots,
$$

which gives

$$
u(x, t)=\frac{1}{x-t^{\prime}}
$$

in the closed form. This solution is exactly the same as we find in (3.35). 


\section{Variational Iteration Method}

To illustrate the basic idea of the variational iteration method (VIM), we consider the following general nonlinear system:

$$
L u(x, t)+N u(x, t)=g(x, t)
$$

where $L$ is a linear operator, and $N$ is a nonlinear operator, and $g(x, t)$ is the source inhomogeneous term.

According to the variational iteration method $[23,38]$, one can construct the following iteration formulation:

$$
u_{n+1}(x, t)=u_{n}(x, t)+\int_{0}^{t} \lambda(t, s)\left(L u_{n}(x, s)+N \tilde{u}_{n}(x, s)-g(x, s)\right) d s,
$$

where $\lambda$ is a general Lagrange's multiplier, which can be identified optimally via the variational theory, and $\tilde{u}_{n}$ is a restricted variation which means $\delta \tilde{u}_{n}=0$.

It is obvious now that the main steps of variational iteration method require first the determination of the Lagrangian multiplier $\lambda$ that will be identified optimally. Having determined the Lagrangian multiplier, the successive approximations $u_{n+1}, n \geq 0$, of the solution $u$ will be readily obtained upon using any selective function $u_{0}$ [38]. Consequently, the solution is obtained as the limit of the resulting successive approximations, that is,

$$
u=\lim _{n \rightarrow \infty} u_{n}
$$

\section{Applications of VIM}

Example 8.1. In this example we solve the initial value problem given by (3.12)-(3.13) using VIM. According to VIM described above, a correction functional for (3.12) can be constructed as follows:

$$
u_{n+1}(x, t)=u_{n}(x, t)+\int_{0}^{t} \lambda(t, s)\left(\frac{\partial u_{n}(x, s)}{\partial s}-\tilde{u}_{n}(x, s)-\frac{\partial^{2} \tilde{u}_{n}(x, s)}{\partial x^{2}}\right) d s,
$$

where $\lambda$ is a Lagrange multiplier, $\tilde{u}_{n}$ is a restricted variation, that is, $\delta \tilde{u}_{n}=0$. To find the optimal value of $\lambda$, we make (8.1) stationary with respect to $u_{n}$, and obtain

$$
\begin{gathered}
\frac{\partial \lambda(t, s)}{\partial s}=0, \\
1+\left.\lambda(t, s)\right|_{s=t}=0 .
\end{gathered}
$$




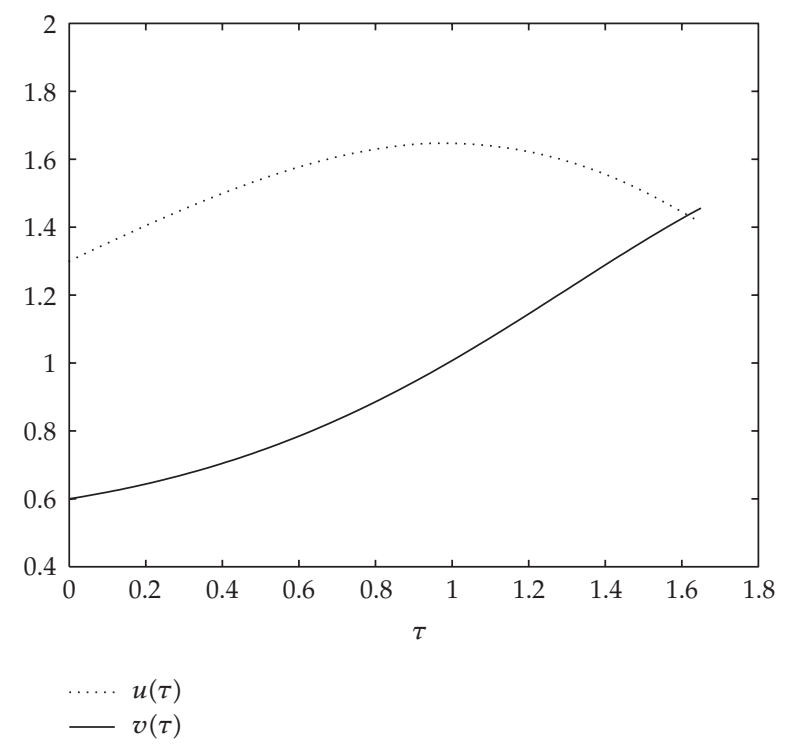

Figure 2: Numerical solutions to the system (1.7) with $\alpha=1, u(0)=1.3, v(0)=0.6$.

The Lagrange multiplier can be identified as $\lambda=-1$, submitting the result into (8.1) leads to the following iteration formula:

$$
u_{n+1}(x, t)=u_{n}(x, t)+\int_{0}^{t}(-1)\left(\frac{\partial u_{n}(x, s)}{\partial s}-u_{n}(x, s)-\frac{\partial^{2} u_{n}(x, s)}{\partial x^{2}}\right) d s, \quad n \geq 0 .
$$

Beginning with an initial approximation $u_{0}(x, t)=u_{0}(x, 0)=\cos (\pi x)$, we obtain the following successive approximations:

$$
\begin{aligned}
u_{1}(x, t) & =\cos (\pi x)+\left(1-\pi^{2}\right) t \cos (\pi x), \\
u_{2}(x, t) & =\cos (\pi x)+\left(1-\pi^{2}\right) t \cos (\pi x)+\frac{1}{2 !}\left(1-\pi^{2}\right)^{2} t^{2} \cos (\pi x), \\
u_{3}(x, t) & =\cos (\pi x)+\left(1-\pi^{2}\right) t \cos (\pi x)+\frac{1}{2 !}\left(1-\pi^{2}\right)^{2} t^{2} \cos (\pi x)+\frac{1}{3 !}\left(1-\pi^{2}\right)^{3} t^{3} \cos (\pi x), \\
\vdots & \\
u_{n}(x, t) & =\cos (\pi x)+\left(1-\pi^{2}\right) t \cos (\pi x)+\frac{1}{2 !}\left(1-\pi^{2}\right)^{2} t^{2} \cos (\pi x)+\cdots+\frac{1}{n !}\left(1-\pi^{2}\right)^{n} t^{n} \cos (\pi x) .
\end{aligned}
$$




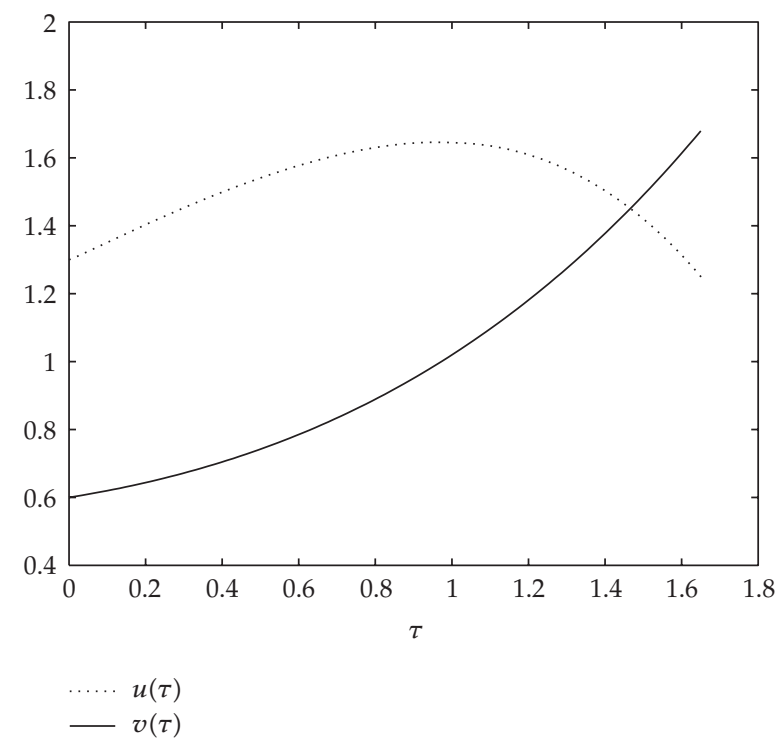

Figure 3: Approximate solutions to the system (1.7) by Adomian's decomposition method with $\alpha=1$, $u(0)=1.3, v(0)=0.6$.

By the use of (7.3), the solution to (3.12)-(3.13) becomes

$$
u(x, t)=e^{\left(1-\pi^{2}\right) t} \cos (\pi x)
$$

which is exactly the same as we have obtained in (3.16).

Example 8.2. In this example we solve the initial value problem given by (3.19) and (3.24) using VIM. For this problem, a correction functional for (3.19) can be constructed as follows:

$$
u_{n+1}(x, t)=u_{n}(x, t)+\int_{0}^{t} \lambda(t, s)\left(\frac{\partial u_{n}(x, s)}{\partial s}+2 \tilde{u}_{n}^{3}(x, s)-\frac{\partial^{2} \widetilde{u}_{n}(x, s)}{\partial x^{2}}\right) d s,
$$

where $\lambda$ is a Lagrange multiplier, $\tilde{u}_{n}$ is a restricted variation, that is, $\delta \tilde{u}_{n}=0$. The optimal value of the Lagrange multiplier is calculated to be $\lambda=-1$ as done in the above example (see (8.2)). Submitting this $\lambda$ into (8.6) leads to the following iteration formula:

$$
u_{n+1}(x, t)=u_{n}(x, t)+\int_{0}^{t}(-1)\left(\frac{\partial u_{n}(x, s)}{\partial s}+2 u_{n}^{3}(x, s)-\frac{\partial^{2} u_{n}(x, s)}{\partial x^{2}}\right) d s, \quad n \geq 0 .
$$




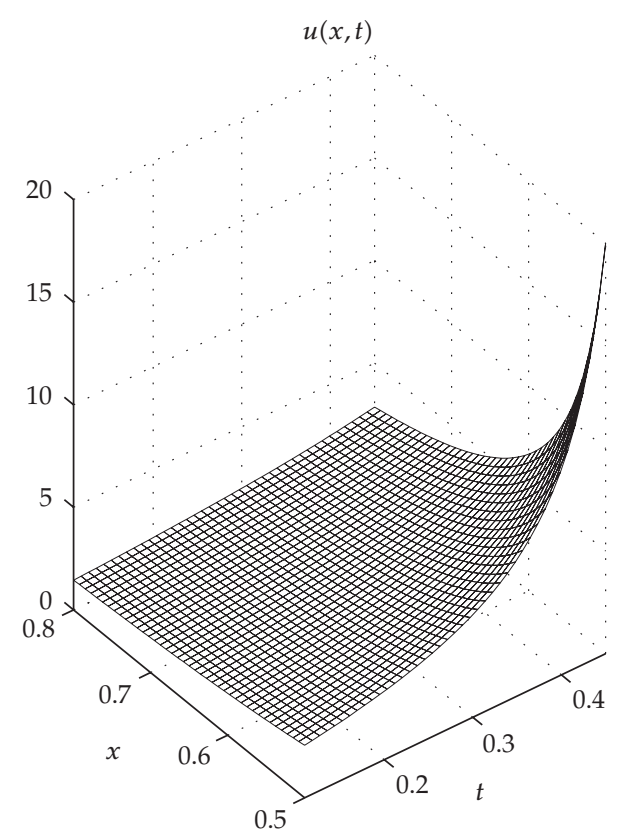

(a)

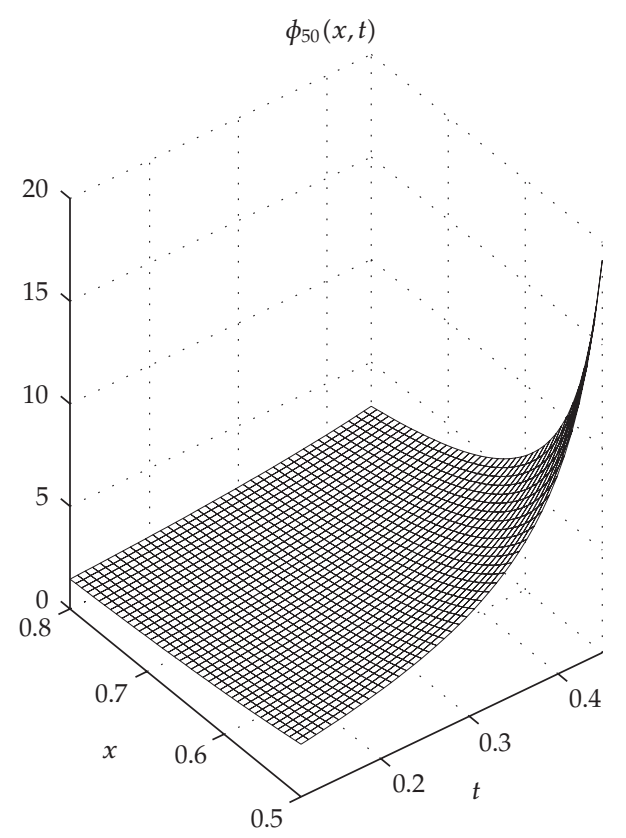

(b)

Figure 4: The numerical results for (3.33), two pictures are almost identical.

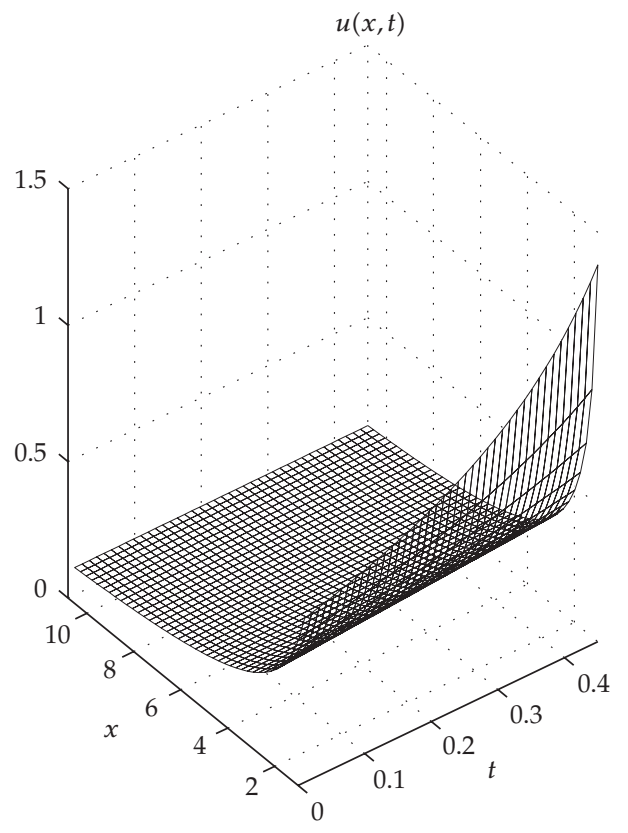

(a)

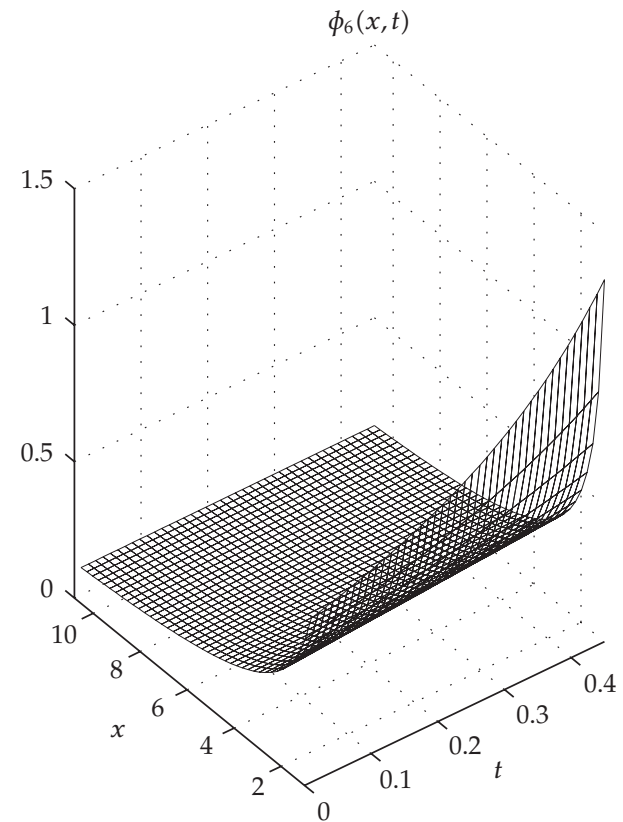

(b)

Figure 5: The numerical results for (3.38), two pictures are almost identical. 
Beginning with an initial approximation $u_{0}(x, t)=u_{0}(x, 0)=(1+2 x) /\left(x^{2}+x+1\right)$, we obtain the following successive approximations:

$$
\begin{aligned}
u_{1}(x, t) & =\frac{1+2 x}{x^{2}+x+1}-\frac{6(1+2 x)}{\left(x^{2}+x+1\right)^{2}} t \\
u_{2}(x, t) & =\frac{1+2 x}{x^{2}+x+1}-\frac{6(1+2 x)}{\left(x^{2}+x+1\right)^{2}} t+\frac{36(1+2 x)}{\left(x^{2}+x+1\right)^{3}} t^{2} \\
u_{3}(x, t) & =\frac{1+2 x}{x^{2}+x+1}-\frac{6(1+2 x)}{\left(x^{2}+x+1\right)^{2}} t+\frac{36(1+2 x)}{\left(x^{2}+x+1\right)^{3}} t^{2}-\frac{216(1+2 x)}{\left(x^{2}+x+1\right)^{4}} t^{3}, \\
& \vdots \\
u_{n}(x, t) & =\frac{1+2 x}{x^{2}+x+1}-\frac{6(1+2 x)}{\left(x^{2}+x+1\right)^{2}} t+\frac{36(1+2 x)}{\left(x^{2}+x+1\right)^{3}} t^{2}-\cdots+\frac{(-1)^{n} 6^{n}(1+2 x)}{\left(x^{2}+x+1\right)^{n+1}} t^{n} .
\end{aligned}
$$

The VIM admits the use of (7.3), therefore, one obtains the exact solution

$$
u(x, t)=\frac{1+2 x}{6 t+x^{2}+x+1}
$$

which is exactly the same as we have obtained in (3.23).

\section{Conclusion and Results}

In this paper we present a review of some recent results for the approximate analytical solutions of nonlinear differential equations. To do this, we introduce and compare three methods, namely, ADM, HPM, and VIM, which are recently studied by the researchers in various fields of science and engineering.

In Section 2, we work out a detailed analysis of ADM for logistic growth model, predator-prey model, for nonlinear heat equation of the form $u_{t}=u_{x x}+u^{m}$, and for the porous media equation, respectively.

In Section 3, we provide several applications of ADM to some nonlinear differential equations of the form mentioned in the last paragraph. For example, Figure 1 shows a very good approximation to the analytical solution of logistic growth model in the time interval $[0,0.4]$ by using only 10 terms of the decomposition series, which indicates that the speed of convergence of this method is very fast. In addition, the overall errors can be made pretty small, and a good approximation to the analytical solution for $\tau \geq 0.4$ can be achieved by adding new terms to the partial sums of the decomposition series given by (3.5).

Figure 2 shows the numerical solutions of the system given by (1.7) with initial data $u(0)=\delta=1.3, v(0)=\beta=0.6$. We obtain these solutions using ode23, an ordinary differential equation solver found in the MATLAB package. Also, Figure 3 shows the approximate solutions to the same system using only 5 terms of the decomposition series. It is clear from the comparison between two figures that there is a very close agreement between the two solutions for both $u$ (prey population) and $v$ (predator population) in the time interval $[0,1.45]$. As mentioned above for the logistic growth model, a very good approximation to the 
numerical solution for $\tau \geq 1.45$ can be achieved by adding new terms to the decomposition series.

Tables 1-2 show the absolute errors $\left|u(x, t)-\phi_{n}(x, t)\right|$, where $u(x, t)$ is the analytical solution of the linear initial-boundary value problem given by (3.12)-(3.14), and $\phi_{n}(x, t)$ are the $n$th partial sums given by (2.25) in $t$ and $x$ directions, respectively. Also, Tables 3-4 show the absolute errors for the nonlinear initial-boundary value problem given by (3.19), (3.24)(3.25) in $t$ and $x$ directions, respectively. For numerical purposes, we take $n=30$ for the linear problem, and take $n=20$ for the nonlinear problem. As seen from Tables 1-2, the absolute errors are very small. The same is also true for the nonlinear problem as it is clear from Tables 3-4. For the nonlinear problem, we achieve a very good approximation to the partial analytical solution by using only 20 terms of the decomposition series, which shows that the speed of convergence of this method is very fast, and the overall errors can be made very small by adding new terms to the series given by (2.25).

In Example 3.5, we directly obtain the analytical solution of the porous media equation (1.10) for $m=1$ using ADM. In Examples 3.6-3.7, the approximate analytical solutions of the porous media equation are obtained for $m=-1$ and $m=-4 / 3$, respectively. The absolute errors have been calculated for these values of $m$ in Tables 5 and 6, respectively. For numerical purposes, we take $n=50$ for $m=-1$, and take $n=6$ for $m=-4 / 3$. As seen from Tables 5 and 6 , the absolute errors in both cases are very small. We do achieve very good approximations to the analytical solutions by using only 50 terms of the decomposition series for the case $m=$ -1 , and by using only 6 terms for the case $m=-4 / 3$. Also, the first pictures of Figures 4 and 5 show the particular analytical solutions for $m=-1$ and $m=-4 / 3$, respectively, whereas the second pictures of Figures 4 and 5 show the corresponding approximate analytical solutions obtained using ADM. In both figures the pictures look almost identical.

On the other hand, in Section 4 we present the idea of HPM which was proposed by Ji-Huan He in 1999. According to this method, the solution is obtained as the summation of an infinite series by constructing a homotopy with an embedding parameter $p \in[0,1]$, which is considered as a "small parameter".

In Section 5, we provide a couple of applications of HPM. For example, in Example 5.1 we apply this method to logistic growth model, and in Example 5.2 we apply it to the Predator-Prey Models. As the reader remembers, we have solved the same problems in Examples 3.1 and 3.2, respectively, using ADM. Even though we obtain the same solutions for both methods, the HPM needs not to calculate Adomian polynomials, and it is very straightforward, and the solution procedure is very simple. Therefore, one clearly can conclude that HPM and He polynomials can completely replace the Adomian method and Adomian polynomials.

Also, in Section 6, we present two applications (Examples 6.1-6.2) of modified HPM to porous media equations. We obtain again exactly the same solutions as we have obtained in Examples 3.5-3.6 using ADM. However, as we have faced in the HPM, the modified HPM is very simple, and we do not have to compute Adomian polynomials in this method either.

In Section 7, we introduce the basic idea of VIM which was proposed by Ji-Huan He. This method is based on the incorporation of a general Lagrange multiplier in the construction of correction functional for the equation. Moreover, we provide a couple of applications of VIM in Section 8. In Examples 8.1 and 8.2, we apply the VIM to the initial value problems which we have solved in Examples 3.3 and 3.4 using ADM. We see that the VIM uses only the prescribed initial conditions and does not require a specific treatment, whereas we do have to compute Adomian polynomials in Examples 3.3 and 3.4 which are tedious (see also Section 2.3). 
As a result, although the numerical results are almost the same, HPM is much easier, and more convenient and efficient than ADM and VIM.

\section{Acknowledgment}

The author would like to thank the referees for their valuable suggestions, which greatly improved the paper.

\section{References}

[1] D. Kaya and A. Yokus, "A numerical comparison of partial solutions in the decomposition method for linear and nonlinear partial differential equations," Mathematics and Computers in Simulation, vol. 60, no. 6, pp. 507-512, 2002.

[2] D. Kaya and M. Aassila, "An application for a generalized KdV equation by the decomposition method," Physics Letters A, vol. 299, no. 2-3, pp. 201-206, 2002.

[3] S. Pamuk, "The decomposition method for continuous population models for single and interacting species," Applied Mathematics and Computation, vol. 163, no. 1, pp. 79-88, 2005.

[4] S. Pamuk, "An application for linear and nonlinear heat equations by Adomian's decomposition method," Applied Mathematics and Computation, vol. 163, no. 1, pp. 89-96, 2005.

[5] S. Pamuk, "Solution of the porous media equation by Adomian's decomposition method," Physics Letters A, vol. 344, no. 2-4, pp. 184-188, 2005.

[6] A.-M. Wazwaz, "The decomposition method applied to systems of partial differential equations and to the reaction-diffusion Brusselator model," Applied Mathematics and Computation, vol. 110, no. 2-3, pp. 251-264, 2000.

[7] G. Adomian, Solving Frontier Problems of Physics: The Decomposition Method, vol. 60 of Fundamental Theories of Physics, Kluwer Academic Publishers, Boston, mass, USA, 1994.

[8] G. Adomian, "A review of the decomposition method and some recent results for nonlinear equations," Computers \& Mathematics with Applications, vol. 21, no. 5, pp. 101-127, 1991.

[9] G. Adomian and R. Rach, "Equality of partial solutions in the decomposition method for linear or nonlinear partial differential equations," Computers $\mathcal{E}$ Mathematics with Applications, vol. 19, no. 12, pp. 9-12, 1990.

[10] G. Adomian, R. Rach, and R. Meyers, "Numerical algorithms and decomposition," Computers $\mathcal{E}$ Mathematics with Applications, vol. 22, no. 8, pp. 57-61, 1991.

[11] G. Adomian, "Solving frontier problems modelled by nonlinear partial differential equations," Computers \& Mathematics with Applications, vol. 22, no. 8, pp. 91-94, 1991.

[12] G. Adomian and R. Rach, "Noise terms in decomposition solution series," Computers $\mathcal{E}$ Mathematics with Applications, vol. 24, no. 11, pp. 61-64, 1992.

[13] G. Adomian, "Explicit solutions of nonlinear partial differential equations," Applied Mathematics and Computation, vol. 88, no. 2-3, pp. 117-126, 1997.

[14] L. Casasús and W. Al-Hayani, "The decomposition method for ordinary differential equations with discontinuities," Applied Mathematics and Computation, vol. 131, no. 2-3, pp. 245-251, 2002.

[15] C. Jin and M. Liu, "A new modification of Adomian decomposition method for solving a kind of evolution equation," Applied Mathematics and Computation, vol. 169, no. 2, pp. 953-962, 2005.

[16] A.-M. Wazwaz and A. Gorguis, "Exact solutions for heat-like and wave-like equations with variable coefficients," Applied Mathematics and Computation, vol. 149, no. 1, pp. 15-29, 2004.

[17] A.-M. Wazwaz, "A reliable technique for solving the wave equation in an infinite one-dimensional medium," Applied Mathematics and Computation, vol. 92, no. 1, pp. 1-7, 1998.

[18] A.-M. Wazwaz, "Adomian decomposition method for a reliable treatment of the Bratu-type equations," Applied Mathematics and Computation, vol. 166, no. 3, pp. 652-663, 2005.

[19] J.-H. He, "Homotopy perturbation technique," Computer Methods in Applied Mechanics and Engineering, vol. 178, no. 3-4, pp. 257-262, 1999. 
[20] A. Ghorbani and J. Saberi-Nadjafi, "Exact solutions for nonlinear integral equations by a modified homotopy perturbation method," Computers \& Mathematics with Applications, vol. 56, no. 4, pp. 10321039, 2008.

[21] D.-H. Shou and J.-H. He, "Beyond Adomian method: the variational iteration method for solving heat-like and wave-like equations with variable coefficients," Physics Letters A, vol. 372, no. 3, pp. 233-237, 2008.

[22] L. M. B. Assas, "Variational iteration method for solving coupled-KdV equations," Chaos, Solitons $\mathcal{E}$ Fractals, vol. 38, no. 4, pp. 1225-1228, 2008.

[23] N. Bildik and A. Konuralp, "The use of variational iteration method, differential transform method and Adomian decomposition method for solving different types of nonlinear partial differential equations," International Journal of Nonlinear Sciences and Numerical Simulation, vol. 7, no. 1, pp. 6570, 2006.

[24] M. A. Noor and S. T. Mohyud-Din, "Variational iteration method for fifth-order boundary value problems using He\&s polynomials," Mathematical Problems in Engineering, vol. 2008, Article ID 954794, 12 pages, 2008.

[25] M. A. Noor and S. T. Mohyud-Din, "Variational iteration technique for solving higher order boundary value problems," Applied Mathematics and Computation, vol. 189, no. 2, pp. 1929-1942, 2007.

[26] M. A. Noor and S. T. Mohyud-Din, "Variational iteration decomposition method for solving higher dimensional initial boundary value problems," International Journal of Nonlinear Science, vol. 7, no. 1, pp. 39-49, 2009.

[27] T. Özis and A. Yıldırım, "Comparison between Adomian's method and He's homotopy perturbation method," Computers \& Mathematics with Applications, vol. 56, no. 5, pp. 1216-1224, 2008.

[28] D. D. Ganji and M. Rafei, "Solitary wave solutions for a generalized Hirota-Satsuma coupled KdV equation by homotopy perturbation method," Physics Letters A, vol. 356, no. 2, pp. 131-137, 2006.

[29] M. Javidi, "Iterative methods to nonlinear equations," Applied Mathematics and Computation, vol. 193, no. 2, pp. 360-365, 2007.

[30] S. T. Mohyud-Din, M. A. Noor, and K. I. Noor, "Some relatively new techniques for nonlinear problems," accepted for Mathematical Problems in Engineering.

[31] E. Yusufoglu, "Variational iteration method for construction of some compact and noncompact structures of Klein-Gordon equations," International Journal of Nonlinear Sciences and Numerical Simulation, vol. 8, no. 2, pp. 153-158, 2007.

[32] J.-H. He, "Homotopy perturbation method for solving boundary value problems," Physics Letters A, vol. 350, no. 1-2, pp. 87-88, 2006.

[33] A. Ghorbani, "Beyond Adomian polynomials: He polynomials," Chaos, Solitons E Fractals, vol. 39, no. 3, pp. 1486-1492, 2009.

[34] A. Ghorbani and J. Saberi-Nadjafi, "He's homotopy perturbation method for calculating Adomian polynomials," International Journal of Nonlinear Sciences and Numerical Simulation, vol. 8, no. 2, pp. 229-232, 2007.

[35] C. Chun, H. Jafari, and Y.-I. Kim, "Numerical method for the wave and nonlinear diffusion equations with the homotopy perturbation method," Computers and Mathematics with Applications, vol. 57, no. 7, pp. 1226-1231, 2009.

[36] S. T. Mohyud-Din, M. A. Noor, and K. I. Noor, "Traveling wave solutions of seventh-order generalized $\mathrm{KdV}$ equations using He's polynomials," International Journal of Nonlinear Sciences and Numerical Simulation, vol. 10, no. 2, pp. 227-233, 2009.

[37] M. A. Noor, K. I. Noor, and S. T. Mohyud-Din, "Modified variational iteration technique for solving singular fourth-order parabolic partial differential equations," Nonlinear Analysis: Theory, Methods $\mathcal{E}$ Applications, In press.

[38] A.-M. Wazwaz, "A comparison between the variational iteration method and Adomian decomposition method," Journal of Computational and Applied Mathematics, vol. 207, no. 1, pp. 129-136, 2007.

[39] M. H. Hojjati and S. Jafari, "Semi-exact solution of elastic non-uniform thickness and density rotating disks by homotopy perturbation and Adomian's decomposition methods—part I: elastic solution," International Journal of Pressure Vessels and Piping, vol. 85, no. 12, pp. 871-878, 2008.

[40] J.-H. He, "Some asymptotic methods for strongly nonlinear equations," International Journal of Modern Physics B, vol. 20, no. 10, pp. 1141-1199, 2006.

[41] J.-H. He, "An elementary introduction to recently developed asymptotic methods and nanomechanics in textile engineering," International Journal of Modern Physics B, vol. 22, no. 21, pp. 3487-3578, 2008.

[42] J.-H. He, "A coupling method of a homotopy technique and a perturbation technique for non-linear problems," International Journal of Non-Linear Mechanics, vol. 35, no. 1, pp. 37-43, 2000. 
[43] J. D. Murray, Mathematical Biology, vol. 19 of Biomathematics, Springer, Berlin, Germany, 2nd edition, 1993.

[44] J. E. Bailey and D. F. Ollis, Biochemical Engineering Fundamentals, McGraw-Hill, Singapore, 2nd edition, 1986.

[45] M. Euler and N. Euler, "Symmetries for a class of explicitly space- and time-dependent $(1+1)$ dimensional wave equations," in Symmetry in Nonlinear Mathematical Physics, Vol. 1, 2 (Kyiv, 1997), pp. 70-78, National Academy of Sciences of Ukraine, Institute of Mathematics, Kiev, Ukraine, 1997.

[46] W. Fushchych and R. Zhdanov, "Antireduction and exact solutions of nonlinear heat equations," Journal of Nonlinear Mathematical Physics, vol. 1, no. 1, pp. 60-64, 1994.

[47] R. M. Cherniha, "New ansätze and exact solutions for nonlinear reaction-diffusion equations arising in mathematical biology," in Symmetry in Nonlinear Mathematical Physics, Vol. 1, 2 (Kyiv, 1997), pp. 138-146, National Academy of Sciences of Ukraine, Institute of Mathematics, Kiev, Ukraine, 1997.

[48] A. D. Polyanin and V. F. Zaitsev, Handbook of Nonlinear Partial Differential Equations, Chapman \& Hall/CRC Press, Boca Raton, Fla, USA, 2004.

[49] T. P. Witelski, "Segregation and mixing in degenerate diffusion in population dynamics," Journal of Mathematical Biology, vol. 35, no. 6, pp. 695-712, 1997.

[50] Y. Cherruault and G. Adomian, "Decomposition methods: a new proof of convergence," Mathematical and Computer Modelling, vol. 18, no. 12, pp. 103-106, 1993.

[51] J.-H. He, "Homotopy perturbation method: a new nonlinear analytical technique," Applied Mathematics and Computation, vol. 135, no. 1, pp. 73-79, 2003.

[52] S. Pamuk and N. Pamuk, "He's homotopy perturbation method for continuous population models for single and interacting species," submitted to Computers and Mathematics with Applications. 


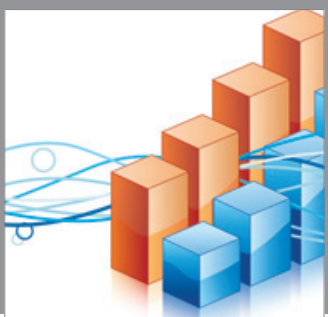

Advances in

Operations Research

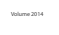

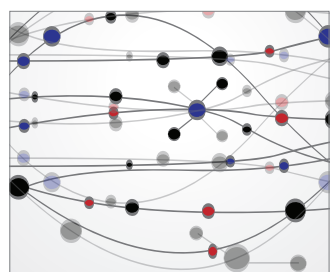

\section{The Scientific} World Journal
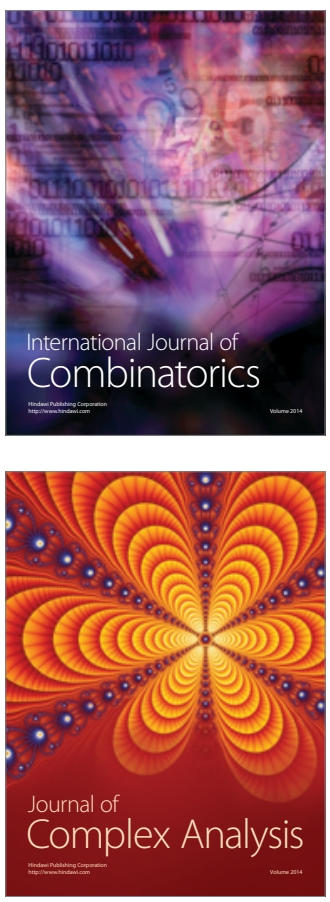

International Journal of

Mathematics and

Mathematical

Sciences
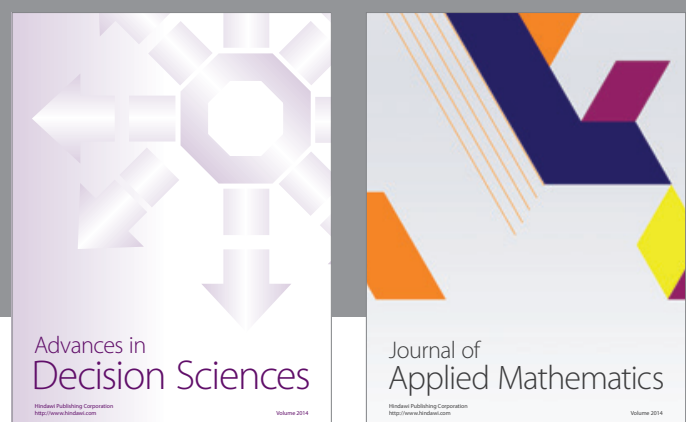

Journal of

Applied Mathematics
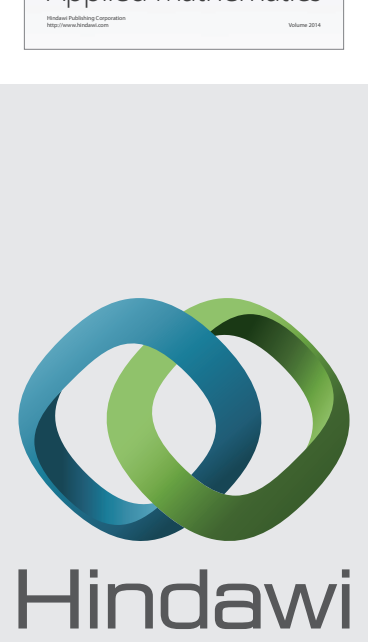

Submit your manuscripts at http://www.hindawi.com
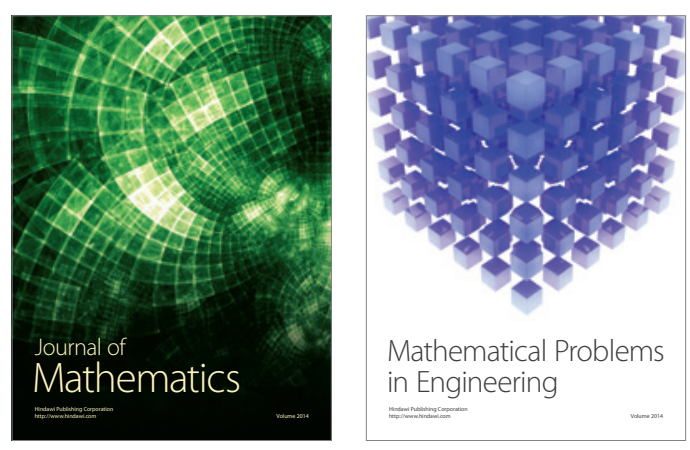

Mathematical Problems in Engineering
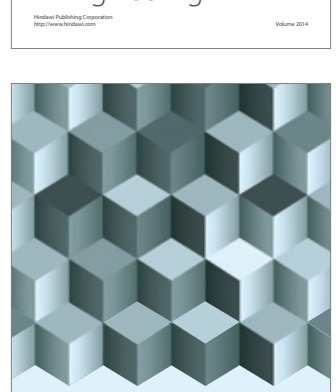

Journal of

Function Spaces
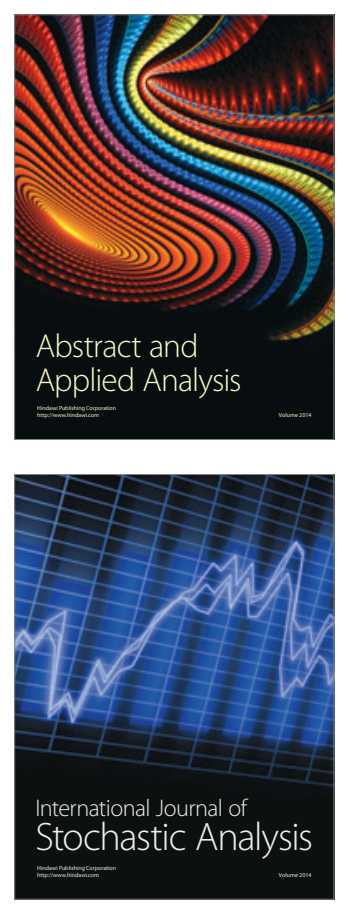

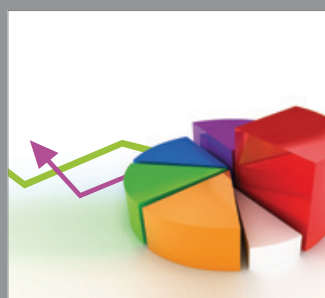

ournal of

Probability and Statistics

Promensencen
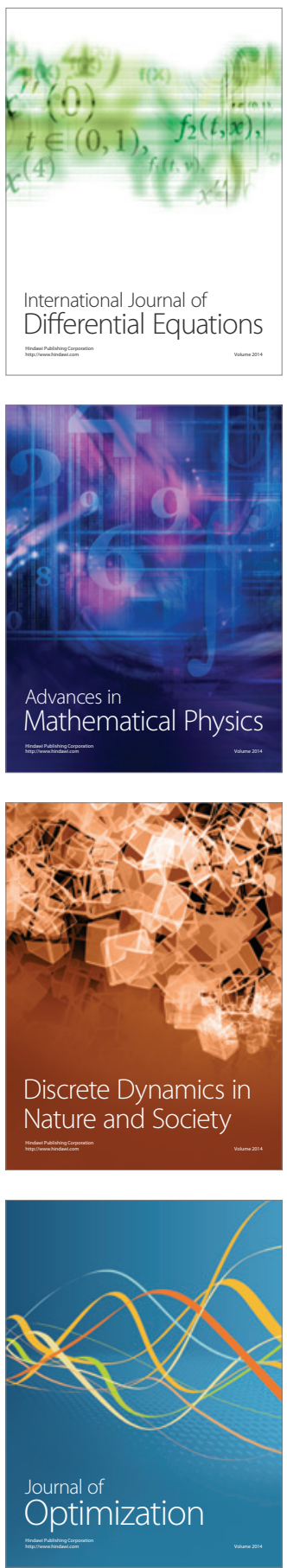